\title{
Integration of III-V lasers on Si for Si photonics
}

\author{
Mingchu Tang ${ }^{1}$, Jae-Seong Park ${ }^{1}$, Zhechao Wang ${ }^{2}$, Siming Chen ${ }^{1}$, Pamela Jurczak ${ }^{1}$, Alwyn \\ Seeds ${ }^{1}$, Huiyun Liu ${ }^{1}$ \\ ${ }^{1}$ Department of Electronic and Electrical Engineering, University College London, Torrington Place, London, WC1E 7JE, \\ United Kingdom \\ ${ }^{2}$ Keysight Laboratories, Keysight Technologies, Inc., 5301 Stevens Creek Boulevard, Santa Clara, California 95051, United \\ States
}

\begin{abstract}
Development of Si photonic integrated circuits (PICs) has been impeded due to lack of efficient Si-based light-emitting sources. Because of their indirect bandgap, bulk Ge and Si are very inefficient at emitting light. Therefore, direct-bandgap III-V semiconductors have been extensively exploited for the active region of the lasers for PICs. Heterogeneous and monolithic integration of III$\mathrm{V}$ semiconductor components on Si platforms have been considered as promising solutions to achieve practical on-chip light-emitting sources for Si photonics. This paper reviews the latest developments on telecommunication wavelength III-V lasers integrated on Si substrates, in terms of integration methods and laser performance.
\end{abstract}

\section{Introduction}

With the dramatic increase of Internet data traffic in recent years, achieving high speed and low-cost optical transmission technology in data centers is of great commercial interest [1-5]. In order to improve the speed of interconnected data transmission, the idea of PICs to integrate semiconductor laser diodes, optical modulators, multiplexers, waveguides, photodetectors, etc. on a single Si chip has emerged [68]. Furthermore, Si photonics integrating PICs or optoelectronic integrated circuits (OEICs) on a silicon platform has attracted a great deal of interest because of the advantages of low cost and large area substrates and compatibility with advanced manufacturing and silicon complementary metal oxide semiconductor (CMOS) fabrication technology [9]. Compared with the state-of-the-art InP-based PICs [10-12], Si-based PICs have been considered as another promising energy efficient solution which could reduce transceiver costs from the current few dollars per gigabit per second $(\mathrm{Gb} / \mathrm{s})$ of input/output $(\mathrm{I} / \mathrm{O})$ bandwidth to less than a few cents per Gb/s [13-15]. Recently, the importance of on-chip optical sources has been emphasized due to reduced packaging costs and higher optical coupling efficiency, even though off-chip light-emitting sources have the advantages of high-temperature performance and high light-emitting efficiency [16]. Moreover, on-chip optical sources offer the potential for dense integration on a single chip and better performance in terms of energy efficiency and scalability.

Despite these advantages, the lack of efficient electrically pumped Si-based light-emitting sources is a key challenge for the commercialization of Si-based PICs. The development of Si-based light-emitting sources is far behind other photonic active and passive components [17-22]. In addition, the Si-based laser has been considered as the "holy grail" of silicon photonics because Si bulk material has an indirect bandgap with very low radiative efficiency [23]. Although demonstrations of Si Raman lasers and Gealloy ( $\mathrm{GeSi}$ and $\mathrm{GeSn}$ ) lasers show that group IV optoelectronic devices integrated on Si platform are possible [24-29], the Raman laser still requires an off-chip light source and the efficiencies of electrically pumped group IV lasers are too low for practical applications. Compared with group IV materials, group III-V materials have superior optical and electrical properties as active components in PICs. For instance, well-established and commercialized GaAs- and InP-based quantum well (QW) or quantum dot (QD) lasers operating at $1.3 \mu \mathrm{m}$ and $1.5 \mu \mathrm{m}$ wavelengths exhibit very low threshold current 
density $\left(\mathrm{J}_{\mathrm{th}}\right)$, long lifetime, fast modulation speed, high output power, small footprint, and high efficiency [30-46]. The QW laser has shown its unique advantages for the fast modulation speed. However, the threshold current and the slope efficiency of QW lasers deteriorates sensitively as temperature increases. Despite there being several demonstrations of QW lasers with high characteristic temperature $\left(\mathrm{T}_{0}\right)$ by using tunneling injection which reduces the hot carrier effects $[47,48]$, the QD laser has a better performance at high temperature than the QW laser, especially using the p-type modulation technique in the QD region [49-55]. Deppe et al. have theoretically predicted a fast directmodulation speed over $30 \mathrm{Gbps}$ by using p-type modulation doping in the QD region [44]. A temperature insensitive operation of $10 \mathrm{Gbps}$ up to $60^{\circ} \mathrm{C}$ and a $25 \mathrm{Gbps}$ direct modulation speed of 1.3 $\mu \mathrm{m}$ QD laser speed has been realized by Sugawara et al. [56]. In this regard, the integration of III-V lasers on Si platform has been recognized to be the desired solution for the Si-based PIC.

There are a few methods for integrating III-V materials on Si substrates: heterogeneous, monolithic and transfer printing. We will focus on reviewing heterogeneous and monolithic integration in this paper, as an extensive review paper on transfer printing can be found in Ref. [57]. Heterogeneous integration is a relatively mature technology to integrate III-V laser on a Si substrate via wafer bonding or flip-chip bonding techniques which overcomes the issue of the different physical properties between Si and IIIV's (GaAs or InP) [58-62]. Compared with heterogeneous integration, however, monolithic integration still needs further efforts to achieve its potential for dense integration.

In this review, we will start by reviewing the recent novel demonstrations of heterogeneous integration of III-V lasers on Si in Section 2. For dense integration of III-V lasers on a Si platform, a variety of light-emitting sources bonded on Si substrates will be presented including a III-V ultra-dense comb sources, a one-dimensional (1D) photonic crystal cavity $(\mathrm{PhC})$ laser, and microring/microdisk cavity lasers. Section 3 discusses the monolithic integration of telecommunications wavelength III-V QD edge-emitting and microdisk/microring lasers, grown on $\mathrm{Ge}, \mathrm{Ge} / \mathrm{Si}$ and $\mathrm{Si}$ substrates with high operating temperature, low threshold current density and long lifetime. In the final section, a brief discussion and comparison of heterogeneous and monolithic integration will be presented.

\section{Heterogeneous integration of III-V lasers on Si substrates}

\subsection{Introduction to bonding techniques}

Heterogeneous integration of III-V lasers on silicon refers to the direct transfer of well-established III$\mathrm{V}$ light-emitting sources to Si wafers while maintaining their high performance. Bonding techniques such as flip-chip and wafer bonding have been extensively explored over the past two decades. Flipchip bonding is used to integrate pre-fabricated optoelectronic devices on $\mathrm{Si}$, with laser structures grown on their native substrates (GaAs or InP mostly). Accurate alignment between III-V lasers and silicon waveguides is a key technology, and the integration density is determined by the pitch density and the size of the bumps [63]. On the other hand, the wafer bonding technique allows wafer-scale processing of devices after bonding III-V thin films on silicon, which enables potentially lower cost, better alignment and high density of integration. Among various bonding techniques, such as direct bonding, anodic bonding, solder bonding [64], eutectic bonding [65], thermocompression bonding [66], direct metal-to-metal bonding, ultrasonic bonding [67], low-temperature melting-glass bonding [68] and adhesive bonding, we will focus on the two most developed technologies: direct bonding and adhesive bonding.

Direct bonding techniques require stringent wafer surface preparation and annealing processes to improve the bonding strength. The manufacturing process must be designed to avoid high-temperature SOI processing $\left(>600{ }^{\circ} \mathrm{C}\right)$, which may damage the III-Vs. A low-temperature annealing method has been investigated by introducing $\mathrm{O}_{2}$ plasma, which is shown in Fig. 1(a) $[69,70]$. The $\mathrm{O}_{2}$ plasma 
facilitates the creation of $\mathrm{OH}$-bonds after the removal of the native oxide layer, which enables $\mathrm{Si}$ and III-Vs to form strong covalent bonds when they mate with each other and are bonded by van der Waals force or hydrogen bonds in the beginning. On the other hand, compared with the direct bonding technology, it was found that adhesive bonding technology using thermosetting polymers as an intermediate layer, such as divinylsiloxane-bis-benzocyclobutene (DVS-BCB), epoxies, spin-onglasses, and polyimides could provide higher bonding strength. The advantages of using DVS-BCB (also referred to as BCB) include high bonding strength and void-free bonds, attributed to low volume shrinkage of the adhesive [61]. Fig. 1(b) shows the process of adhesive bonding of III-V dies on a Si substrate by using bonding agent DVS-BCB [71]. The whole adhesive bonding process does not require high-temperature operation, and hence reduces the risk of III-V layer damage.

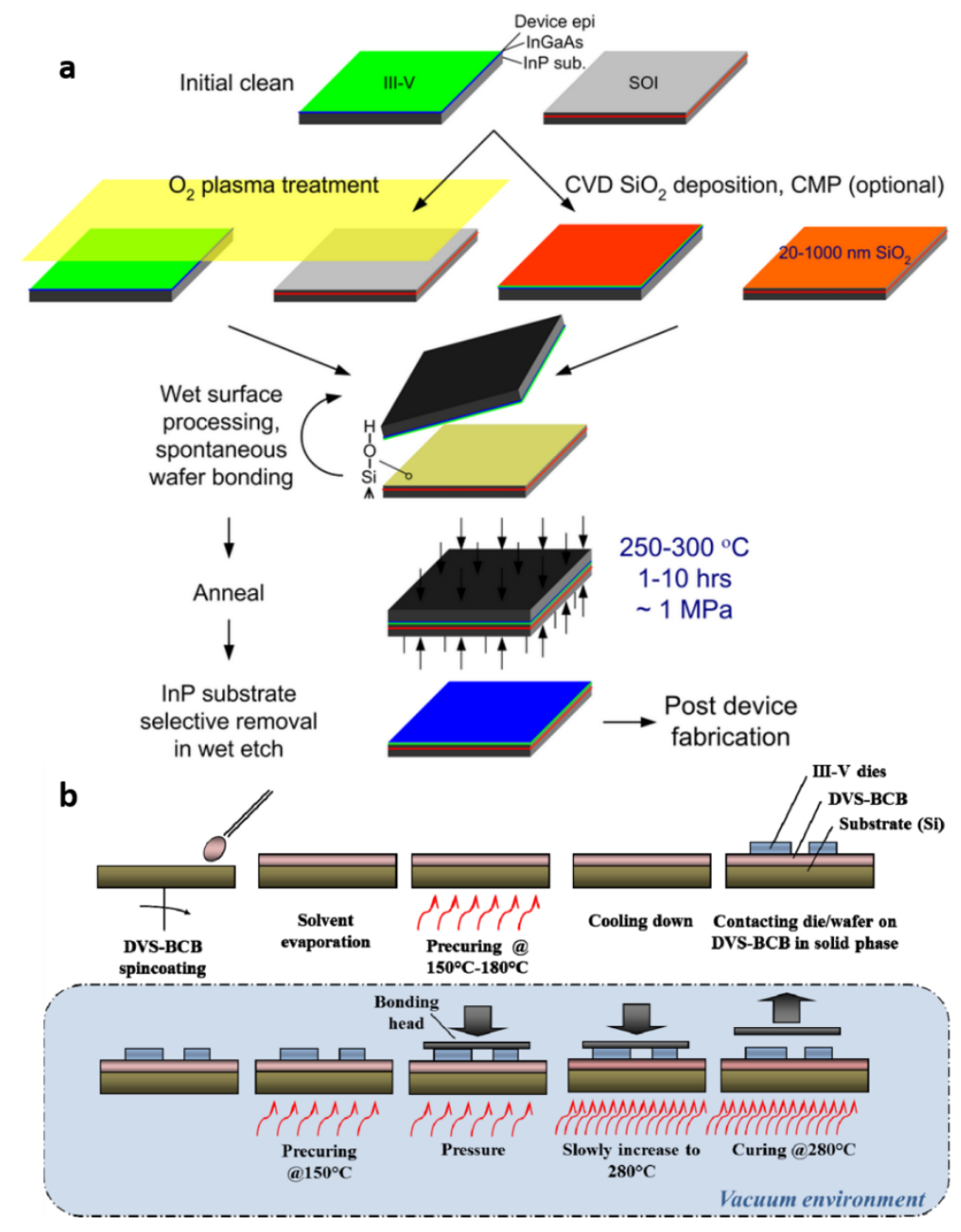

Fig. 1. Process flow of, (a), $\mathrm{O}_{2}$ plasma assisted direct bonding of InP on SOI; (b), die-to-die/wafer adhesive bonding via DVS-BCB. Reproduced from reference $[62,71]$.

Employing direct and adhesive bonding technologies, there are many examples of III-V lasers bonded to Si substrates. For example, a $1 \mu \mathrm{m}$ wavelength InGaAs/GaAs QW laser bonded to a Si substrate was achieved in 1992 [72]. InGaAsP/InP and InGaAs/InP QW lasers with longer wavelength emission bonded to Si substrates were demonstrated afterward [73, 74]. From 2005 to 2008, researchers from the University of California, Santa Barbara (UCSB) demonstrated a series of InAlGaAs QW lasers on Si emitting in the telecommunication wavelength region around 1.3 and $1.5 \mu \mathrm{m}$ [60, 75-82]. For instance, Fang et al. established a method for integrating high volume III-V lasers on Si. As shown in Fig 2(a), the optical mode is overlapped within the Si waveguide and the III-V laser. At the same time, researchers from III-V Lab, CEA Leti, France, Ghent University, Belgium, and Eindhoven University 
of Technology, Netherlands reported their intensive work on InP lasers bonded to Si by using adhesive bonding technology [83-90]. In 2006, Roelkens et al. showed InGaAsP QW laser and photodetector bonding on a Si substrate via DVS-BCB, by introducing an inverted adiabatic taper to increase the coupling efficiency from a Si waveguide to the III-V mesa, as shown in Fig. 2(b). A very recent demonstration of InP DFB laser bonding on SOI with ultrafast modulation speed of $56 \mathrm{~Gb} / \mathrm{s}$ (direct and electro-absorption modulation) was published by Abbasi et al., which exceeded the bandwidth of commercially available InP modules (40Gb/s) [91]. A series of studies on InAs/GaAs QD lasers on $\mathrm{Si}$ substrates using wafer bonding techniques have been reported by researchers from Tokyo University since 2009 [92-96]. Recently, direct modulation of a QD laser wafer bonded on a Si substrate with speed of $10 \mathrm{~Gb} / \mathrm{s}$ was demonstrated in a collaboration between Tokyo University and QD laser Inc. [59].
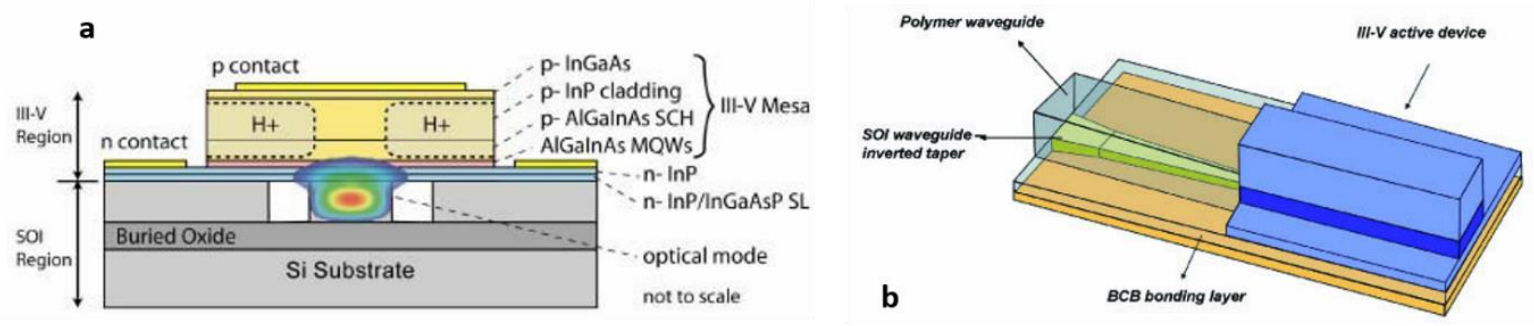

Fig. 2. Schematic diagram of (a), AlGaInAs MQW laser on $\mathrm{Si}$ by using low-temperature $\mathrm{O}_{2}$ plasma assisted bonding technology and (b), InGaAsP laser bonding on SOI by using adhesive bonding technology. Reproduced from references $[60,63]$.

\section{$2.2 \quad$ III-V-on-Si ultra-dense comb lasers}

Mode-locked lasers (MLLs) generate discrete frequency combs spaced by the longitudinal cavity mode spacing. Such optical combs find wide applications in the fields of frequency metrology, optical clocks [97], and spectroscopy [98]. Moreover, high speed wavelength division multiplexing (WDM) also benefits from using such a multi-wavelength source [99]. Compared with traditional fiber-based MLLs, integrated MLLs have the advantages of small form factor, lower cost and comb line spacings suitable for dense WDM operation. Heterogeneously integrated MLLs have also been widely explored in recent years. Koch et al. demonstrated an MLL bonded to a Si substrate with a repetition rate up to $40 \mathrm{GHz}$ and the generation of 4 ps pulses by the design of a two-section Fabry-Perot (F-P) cavity in 2007 [80]. A racetrack resonator was also employed to enable mode-locking with a repetition rate of $30 \mathrm{GHz}$ and a pulse width of $7 \mathrm{ps}$ [100]. An InAs/GaAs QD MLL based on a GaAs laser bonded to Si was developed by Wang et al., showing a repetition rate of $7.3 \mathrm{GHz}$ and a pulse of about 2 ps width [101].

The key advantage of heterogeneously integrating MLLs on Si is that one can increase the photon lifetime by leveraging low loss silicon waveguides. The large bandgap passive silicon waveguide can also serve as a building block of a long external cavity that effectively reduces the laser linewidth (and phase noise) substantially. Wang et al. recently demonstrated a III-V QW MLL bonded on silicon, which shows exceptional performance [58]. As shown in Fig. 3(a) and (b), the MLL includes a long Si spiral waveguide $(37.4 \mathrm{~mm})$ with low loss $(0.7 \mathrm{~dB} / \mathrm{cm})$, two optical amplifiers separated by a saturable absorber, and two distributed Bragg reflectors (DBR) acting as the mirrors of the cavity $[102,103]$. The III-V part was bonded on SOI via a 30-nm thick DVS-BCB bonding layer. The $40 \mu \mathrm{m}$ length saturable absorber was etched and consequently isolated with two gain sections, which are separated by a $15 \mu \mathrm{m}$ gap. The resultant laser is passively mode-locked at $1 \mathrm{GHz}$, generating a 10-dB optical bandwidth that spans over $15 \mathrm{~nm}$ with more than 1400 phase-locked lines as shown in Fig. 3(c). In Fig. 4(a), the RF 
spectrum of the generated pulse train is presented. The strong RF fundamental tone and the clean spectrum in the low-frequency range (inset) indicate stable mode-locking and low residual noise modulation. From Fig. 4(b), the 10-dB linewidth measured is found to be below $900 \mathrm{~Hz}$, which is one of the lowest among the demonstrated integrated MLLs in the literature. The optical linewidth of the MLL is also very low, which is in the range of below $250 \mathrm{kHz}$. These results proved a successful integration of passive waveguide and MLL on SOI platform with low repetition rate $(1 \mathrm{GHz})$ and large optical bandwidth (12 $\mathrm{nm}$ passive and $13 \mathrm{~nm}$ Hybrid locked).

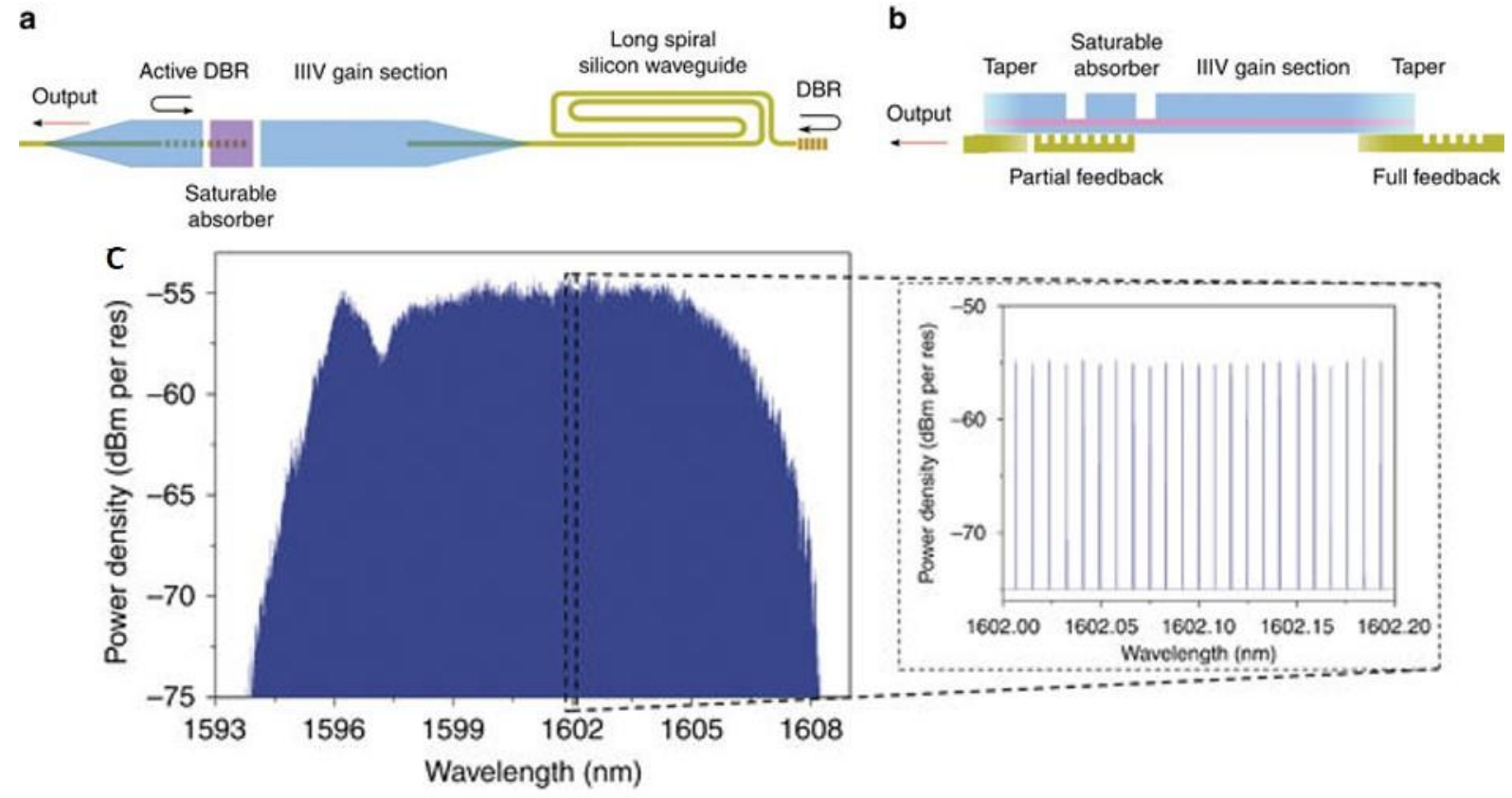

Fig. 3. Layout of III-V comb laser heterogeneously integrated on SOI for (a) top view, and (b) side view. (c) Output spectrum when the laser is passively locked at $1 \mathrm{GHz}$ repetition rate. Reproduced from reference [58].
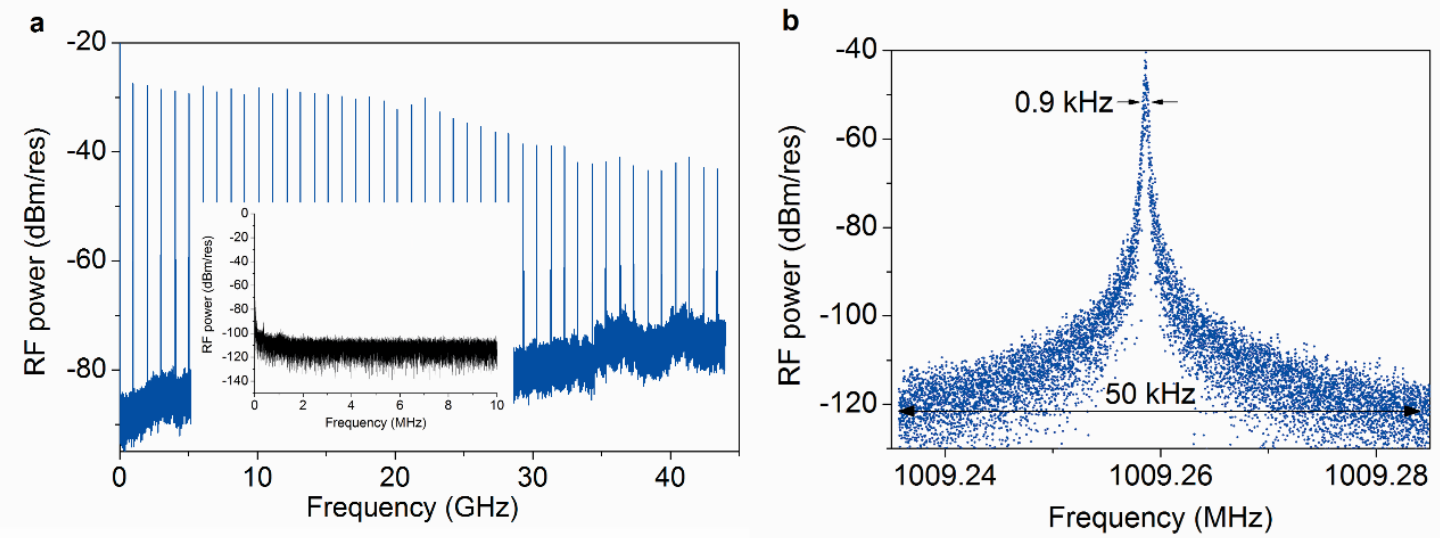

Fig. 4. (a) RF spectrum of the generated optical pulse train. Inset: enlarged RF spectrum in the frequency range of 1-10 MHz. (b) Detail of the 1-GHz RF tone. Reproduced from reference [58].

\subsection{Photonic crystal nanocavity lasers}

Photonic crystal $(\mathrm{PhC})$ cavity lasers have a number of advantages, such as low power consumption, high modulation speed and small footprint $[42,104,105]$. While high-dimensional $\mathrm{PhC}$ lasers bonded 
on Si substrates have been extensively studied [95, 106-109], the one-dimensional system has its unique properties including the provision of the singular density of states and the possibility for photon transport within the 1D photonic bands [110].

Crosnier et al. recently reported their progress on the integration of $\mathrm{InP} \mathrm{PhC}$ lasers on $\mathrm{Si}$ via the adhesive bonding technique [111]. As illustrated in Fig. 5(a) and (b), the 1D PhC laser is formed by a long III-V rib waveguide of $15 \mu \mathrm{m}$ in length bonded on top of a $220 \times 550 \mathrm{~nm}^{2}$ SOI waveguide. Electrical injection of nanolasers is very difficult due to the limited laser size for electrode attachment and the high optical loss introduced by the proximity of the electrodes to the optical mode [112]. In this work, the authors engineered the nanorib design to enable highly efficient current injection without spoiling its optical properties. The fabricated laser was tested in continuous-wave (CW) mode at room temperature (RT), showing a single-mode emission at $1.56 \mu \mathrm{m}$ with over $60 \mathrm{~dB}$ side-mode-suppression-ratio, as shown in Fig. 5(c). Fig. 5(d) presents the L-I-V measurement results of the laser. The series resistance and turnon voltage were measured to be $1.8 \mathrm{k} \Omega$ and $0.75 \mathrm{~V}$, respectively. The measured threshold current was $100 \mu \mathrm{A}$ at an applied voltage of $1 \mathrm{~V}$, with a max output power of $95 \pm 2 \mu \mathrm{W}$. This work clearly shows the advantages of high integration density, with a record performance of high wall-plug and differential quantum efficiencies, which are $14 \%$ and $35 \%$, respectively $[109,113,114]$.
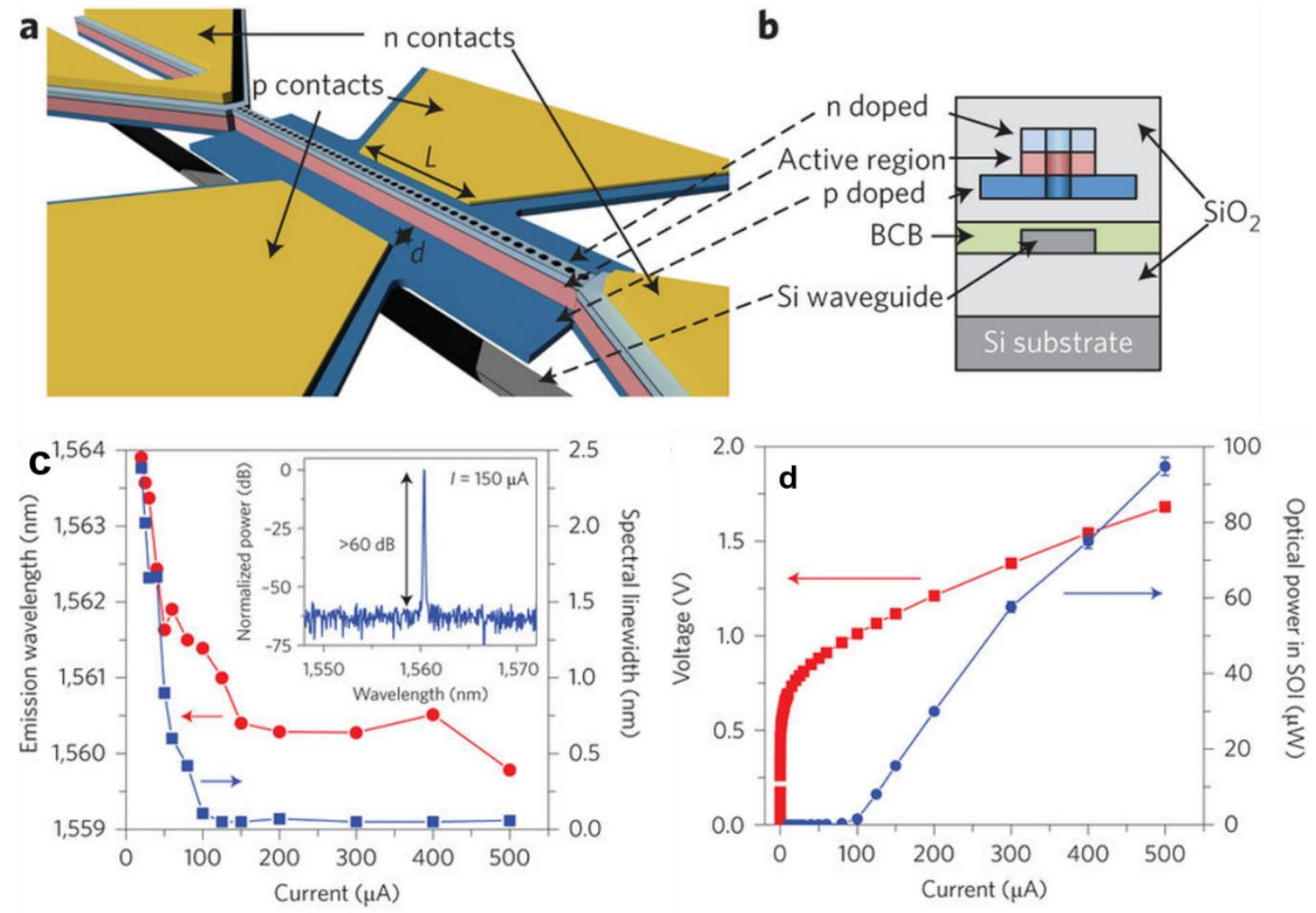

Fig. 5. 1D InP PhC nanolaser bonded on Si. Schematic diagram of the laser structure with (a) 3dimension view and (b), Cross-section of the nanorib structure bonded on Si waveguide via BCB. (c) Emission wavelength and spectral linewidth against injection current at room temperature; inset: lasing spectrum at an injection current of $150 \mu \mathrm{A}$. (d) L-I-V measurements of the nanolaser at room temperature. Reproduced from reference [111].

\subsection{Microdisk and microring lasers}


Microdisk and microring lasers bonded on Si have been widely explored over the last decade. Compared with conventional F-P lasers, microdisk and microring lasers with few micrometer diameters have much lower cavity losses, facilitating low threshold operation. In particular, the bending loss of micrometer microdisk and microring resonator is normally very low, given the high refractive index contrast between the disk and the surrounding medium (air) [115]. It is also straightforward to out-couple the light due to their inherent traveling wave operation nature [116]. Moreover, circular resonators provide a smaller footprint than that of F-P cavities, which could increase the integration density. The first demonstration of CW operation of an InP-based microdisk laser bonded on Si was reported by Seassal et al. in 2001 [117]. In 2007, researchers from Ghent University developed a new integration method for coupling a microdisk laser to a Si waveguide buried under the III-V region [87]. Later, Liang et al. reported a self-aligned deep etching process, which enables small bending radius and low power laser operation [118].

While microring and microdisk resonators have the advantage of high cavity $\mathrm{Q}$, their practical application in a photonic link is hindered by their susceptibility to fabrication imperfections and environmental factors. Additional power is required to tune and stabilize the output signal integrity. Recently, Liang et al. reported a tunable microring laser bonded on $\mathrm{Si}$, integrated with a Si metal-oxidesemiconductor (MOS) capacitor for fast and efficient laser tuning using the plasma-dispersion effect [119]. As shown in Fig. 6(a) and (b), a bonded microring laser is coupled with a Si bus waveguide. The laser is operated by injecting current through terminals $\mathrm{N}$ and $\mathrm{P} 1$, where a third terminal, $\mathrm{P} 3$, is designed to supply bias voltage through terminal $\mathrm{N}$. The MOS capacitor is formed by a dielectric layer embedded between the Si waveguide and the n-InP layer. In Fig. 6(c), the dielectric layer composed of interface oxide is formed as the by-product of the low temperature $\mathrm{O}_{2}$ plasma-assisted bonding process. The free carrier concentration between terminals $\mathrm{N}$ and $\mathrm{P} 1$ can be controlled by changing the applied voltage on the terminal $\mathrm{P} 2$ and $\mathrm{N}$, inducing the variation of the free carrier absorption loss and refractive index on $\mathrm{Si}$ and n-InP. As shown in Fig. 6(d) and (e), the bias voltage of the MOS capacitor affects the photocurrent and lasing wavelength, which functions as a highly efficient tunable laser.
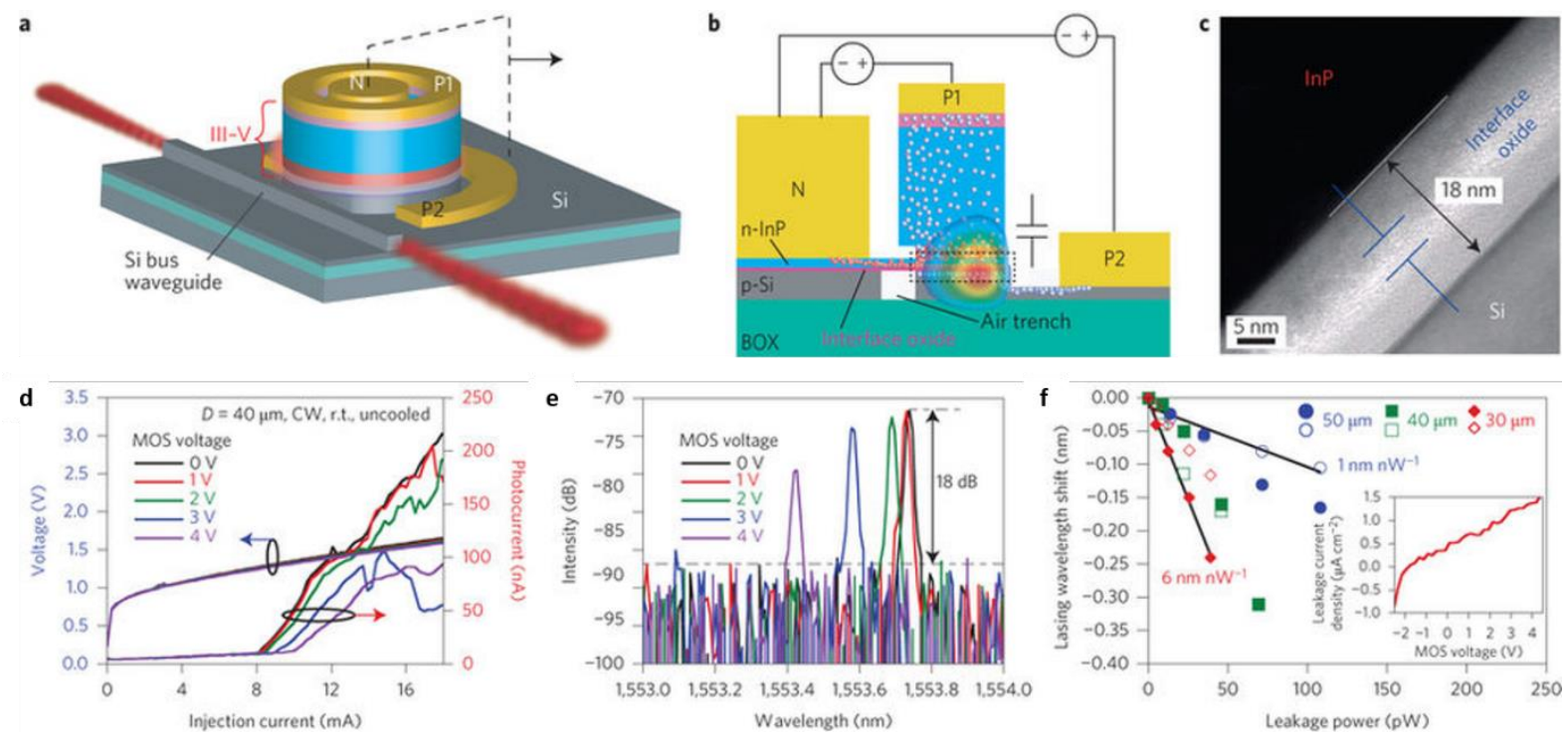

Fig. 6. InP microring laser bonding on $\mathrm{Si}$ substrate and adjacent to a $\mathrm{Si}$ bus waveguide. (a) 3dimensional schematic diagram and (b) cross-section diagram of the laser with MOS capacitor. (c) TEM image of a bonded InP-to-Si with a buried oxide layer. (d) L-I-V measurement and (e), spectra of emission of the laser at different MOS voltage. (f) Lasing wavelength versus leakage power between the terminals $\mathrm{P} 2$ and $\mathrm{N}$ in devices with different diameters; inset: leakage current density versus MOS capacitor bias voltage. Reproduced from reference [119] 


\section{Monolithic integration of III-V lasers on Si substrates}

\subsection{Introduction}

Although bonding techniques have brought the opportunity to integrate numbers of III-V lasers on $\mathrm{Si}$ substrates, monolithic integration has been considered as another promising solution to integrate III-V lasers on Si with dense integration density. Monolithic integration provides a technique to grow III-V compound semiconductor materials on group IV substrates, using molecular beam epitaxy (MBE), chemical vapor deposition (CVD) or vapor phase epitaxy (VPE). The main challenges of monolithic integration are the anti-phase domains (APDs), dislocations and thermal cracks due to the difference in the physical properties between III-V materials and group IV materials. The different polarity of III-V and group IV material can cause APDs. The lattice mismatch between III-V and IV materials, for instance, 4\% lattice mismatch between $\mathrm{GaAs}$ and $\mathrm{Si}$, can introduce a high density of threading dislocations $\left(\sim 10^{10} \mathrm{~cm}^{-2}\right)$ which degrades the laser performance by creating a dangling bond and nonradiative recombination centers. The mismatch of thermal expansion coefficient can lead to the formation of micro-cracks on the epi-materials during the cooling down from high temperature $\left(>500{ }^{\circ} \mathrm{C}\right)$. Until now, in order to reduce the threading dislocation density to the order of $10^{6} \mathrm{or}^{10^{5} \mathrm{~cm}^{-}}$ ${ }^{2}$, many researchers have adopted methods using strained epi-layers as defects filter layers (DFLs). For the issue of APDs, it was found that APDs could be solved by growing III-V materials on group IV oriented substrates from (100) to [110] or [111] with different degree angles from $4^{\circ}$ to $7^{\circ}[120,121]$. However, to avoid the use of offcut substrate incompatible with current Si CMOS technology, it is imperative to grow III-V materials on exact (100) Si substrates. In this regard, GaAs and GaP nucleation layer grown on Si exact (100) substrate by using CVD or VPE have been carried out recently, which brings possibilities to grow III-V laser structures on those platforms. At the same time, the solution of using a patterned Si substrate on which GaAs or $\mathrm{InP}$ was grown has been proposed with the possibility of its low threading dislocation density which could increase the laser performance theoretically.

The development of III-V semiconductor lasers monolithically grown on Si substrates has been pursued for 30 years as shown in Fig. 7, since the first double-heterostructure GaAs/AlGaAs laser grown on $\mathrm{Si}$ working at $77 \mathrm{~K}$ was established in 1984 by Windhorn et al.[122]. Room temperature operation was achieved one year later by the same group from MIT [123]. Almost at the same time, Sakai et al. had demonstrated an $\mathrm{AlGaAs} / \mathrm{GaAs} \mathrm{DH}$ laser on $\mathrm{Si}$ with lower $\mathrm{J}_{\text {th }}\left(4.9 \mathrm{kA} / \mathrm{cm}^{2}\right)$ by using a $\mathrm{GaP}$ buffer layer [124]. Soon, a large number of studies on single-quantum well (SQW) lasers and multiple-quantum well (MQW) lasers grown on Si substrates were published in 1986 and 1987. First, Fischer et al. demonstrated an AlGaAs/GaAs SQW laser with a $\mathrm{J}_{\text {th }}$ of $6.9 \mathrm{kA} / \mathrm{cm}^{2}$ at $7{ }^{\circ} \mathrm{C}$ [125]. Dupuis and his colleagues van der Ziel et al. successfully decreased the $\mathrm{J}_{\text {th }}$ down to $3.5 \mathrm{kA} / \mathrm{cm}^{2}$ and achieved continuous operation $(\mathrm{CW})$ up to $170 \mathrm{~K}$, attributed to the introduction of $\mathrm{GaAsP} / \mathrm{GaAs}$ strained-layer superlattices (SLSs) [126]. Deppe et al. had improved the GaAs buffer layer on a Si substrate by introducing a lowtemperature nucleation layer and thermal cycling on the growth of GaAs, allowing room temperature $\mathrm{CW}$ operation to be achieved [127-130]. It should be noted that until Razeghi et al. published a $1.3 \mu \mathrm{m}$ MQW laser on Si $[131,132]$, all the lasers mentioned above were working at wavelengths shorter than $1 \mu \mathrm{m}$, due to the use of GaAs bulk and AlGaAs/GaAs quantum well structure as a gain medium [133]. The realization of $1.3 \mu \mathrm{m}$ lasers meant that the laser on $\mathrm{Si}$ could be used as a telecommunication and inter-chip connection. More than 2,000 hours' aging tests of $1.5 \mu \mathrm{m}$ QW lasers grown on $\mathrm{InP} / \mathrm{Si}$ substrates were performed by Sugo et al. in 1992, by growing a very thick InP buffer layer $(12 \mu \mathrm{m})$ on $\mathrm{Si}$ [134, 135]. Recently, researchers from the University of Montpelier have demonstrated $1.56 \mu \mathrm{m}$ wavelength lasers with an active region of $\mathrm{Ga}_{0.8} \mathrm{In}_{0.2} \mathrm{Sb}$ QWs separated by $20 \mathrm{~nm} \mathrm{Al} \mathrm{Al}_{0.35} \mathrm{Ga}_{0.65} \mathrm{As}_{0.03} \mathrm{Sb}_{0.97}$ barriers grown on large lattice mismatched $\mathrm{GaSb} / \mathrm{Si}$ virtual substrate (13\%), which operated under $\mathrm{CW}$ mode up to $45^{\circ} \mathrm{C}$ with threshold current density $5 \mathrm{kA} / \mathrm{cm}^{2}$ at room temperature $[136,137]$. 
Together with the advances on QW lasers, the concept of the QD laser was first developed by Arakawa and Sakaki in 1982 [138], and the first QD lasers grown (GaAs island/QD) on a Si substrate was realized by Egawa et al.[139]. Compared with QW lasers on Si substrates, QD lasers have the advantages of low $\mathrm{J}_{\text {th }}$ and high-temperature performance due to the delta-function-like density of states. In addition, a QD laser exhibits better performance on lifetime because of its strong tolerance of threading dislocations. As shown in Fig. 8, threading dislocations (TDs) would either go through and kill the QD or bypass the QD and be bent by the QD due to its strain fields. Linder et al. demonstrated $1 \mu \mathrm{m}$ wavelength $\mathrm{In}_{0.4} \mathrm{Ga}_{0.6} \mathrm{As}$ QD laser grown on Si requiring very low-temperature operation of $80 \mathrm{~K}$ and high $\mathrm{J}_{\text {th }}$ of $3.85 \mathrm{kA} / \mathrm{cm}^{2}$ in 1999 [140]. In 2001, Kazi et al. reported InGaAs QD-like lasers grown on $\mathrm{Si}$ substrates emitting around $850 \mathrm{~nm}$ wavelength [141]. Soon, Mi and Yang et al. investigated the effect of QD dislocation filter layer on the $1.1 \mu \mathrm{m}$ wavelength InAs QD laser grown on Si substrate [142-144]. Based on the concept of dislocation filter layer (DFL), researchers from University College London (UCL) have developed SLSs as DFL and successfully reduced the threading dislocation density down to the order of $10^{6} \mathrm{~cm}^{-2}$, leading to further improvements in operating current and temperature. Moreover, the laser devices are emitting at 1.3- $\mu \mathrm{m}$ wavelength range [145-148]. In the meantime, highperformance QD lasers grown on Ge and Ge/Si substrates have been demonstrated by UCL and UCSB with low threshold current density and long lifetime [149-153].

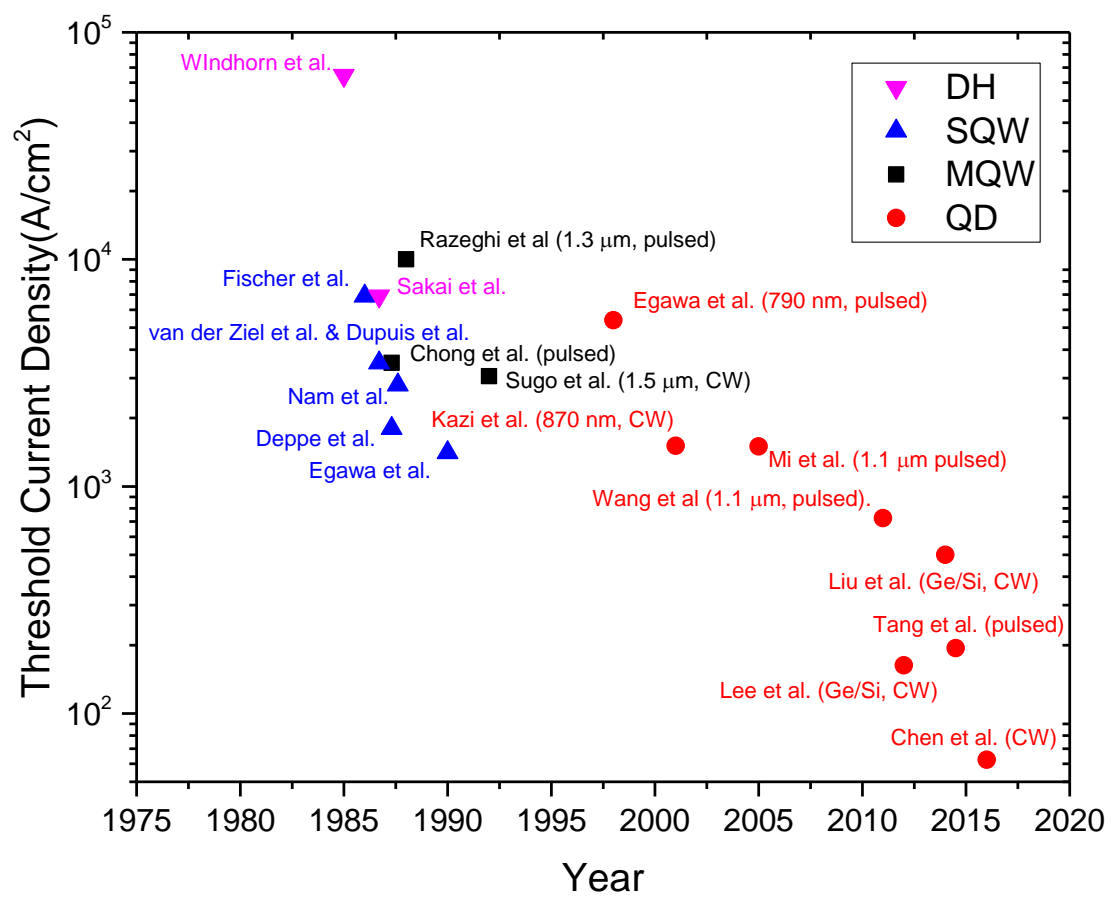

Fig. 7. The historical development of heterostructure lasers monolithically grown on Si substrate, showing the recorded threshold current density. 

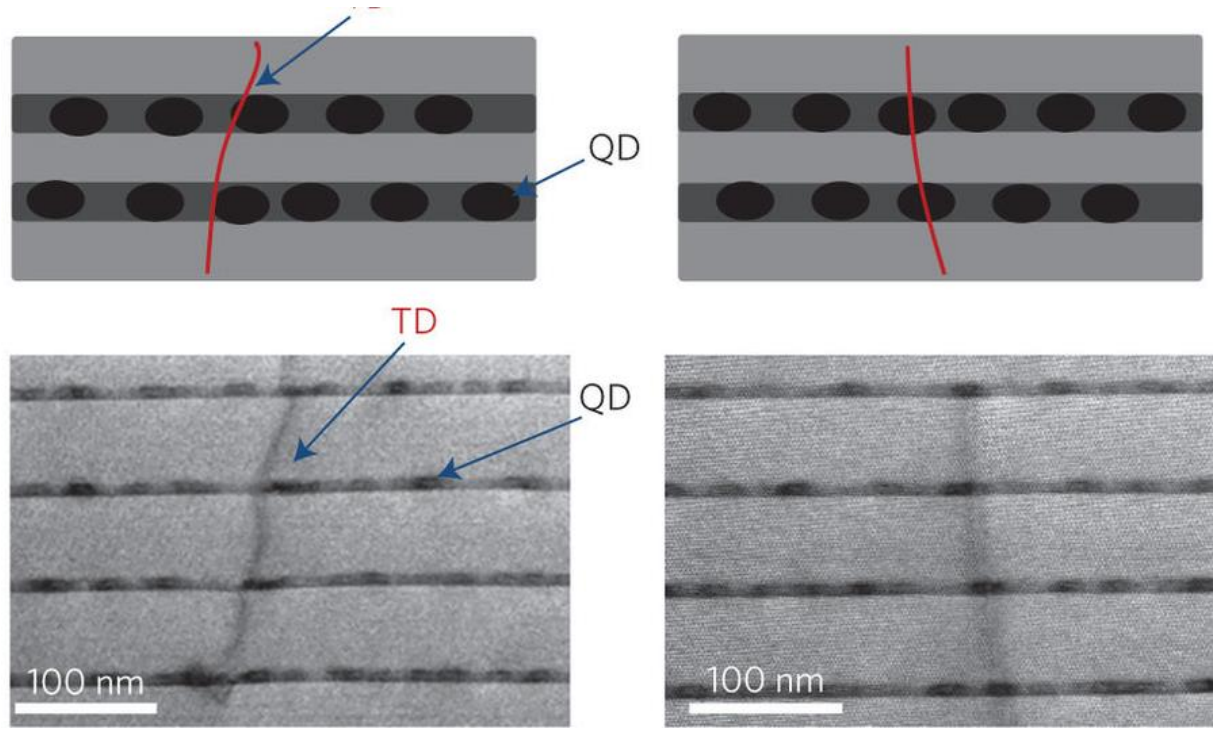

Fig. 8. Schematic diagram and bright-field TEM images of two situations of TD propagating into QD layers. Reproduced from reference [147].

\subsection{InAs/GaAs QD Lasers Grown on $\mathrm{Ge}$ and $\mathrm{Ge} / \mathrm{Si}$ substrates}

A Ge substrate has the advantage of small lattice mismatch between GaAs and Ge. Therefore, highquality GaAs materials can be epitaxially grown on a Ge substrate to achieve the integration of III-V nMOS and Ge pMOS [154]. Accordingly, intensive efforts have been made to grow GaAs on Ge substrates and, consequently, integrating QD lasers on Ge substrates achieved similar lasing performance to QD lasers grown on native GaAs substrates. For example, Liu et al. reported the first demonstration of a $1.3 \mu \mathrm{m} \mathrm{InAs/GaAs}$ QD laser monolithically grown on a Ge substrate in 2011 using an MBE system [149], where a Ga pre-layer technique was employed in the initial growth stage of GaAs on Ge (100) substrate oriented to [111] with 6-degree offcut, in order to achieve single domain GaAs nucleation layer on Ge substrates. In Fig. 9(a) and (b), based on atomic force microscope (AFM) analysis, it is clearly visible that the Ga pre-layer showed remarkably better morphology than that of As pre-layer. Moreover, unlike the As pre-layer technique, the transmission electron microscope (TEM) images indicate that the defects were effectively trapped within $150 \mathrm{~nm}$ of GaAs grown on Ge substrate with the Ga pre-layer technique, as shown in Fig. 9(c) and (d). Consequently, it was shown that the performance of lasers fabricated with a Ga pre-layer was significantly improved. As shown in Fig. 9(e), a maximum operating temperature of $60^{\circ} \mathrm{C}$ was obtained under continuous-wave $(\mathrm{CW})$ operation mode with low $\mathrm{J}_{\mathrm{th}}$ of $55.2 \mathrm{~A} \mathrm{~cm}^{-2}$, corresponding to $11 \mathrm{~A} \mathrm{~cm}^{-2}$ for each of the five QD layers. These results are comparable to the QD lasers grown on GaAs substrates. Also, Wang et al. investigated the effect of the growth temperature of the GaAs nucleation layer on the performance of 1.3- $\mu \mathrm{m}$ QD laser grown on Ge substrate and achieved a QD laser with a room temperature threshold current density of $106 \mathrm{~A} / \mathrm{cm}^{2}$ with operation temperature up to $100{ }^{\circ} \mathrm{C}$ [155]. Also, a further study of an InAs/GaAs QD superluminescent diode grown on Ge substrate was investigated by Jiang et al. [156]. 

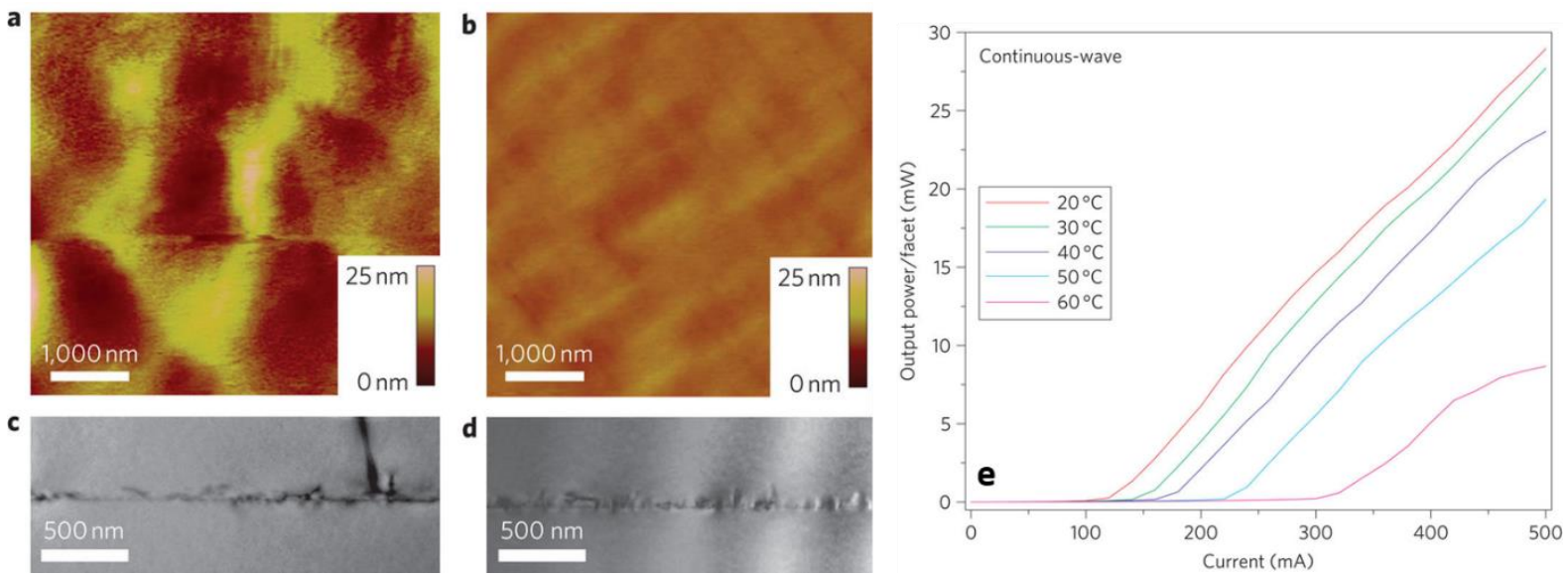

Fig. 9. AFM images of GaAs grown on Ge substrate with (a) As prelayer and (b) Ga prelayer. Crosssection TEM images of GaAs grown on Ge substrate with (c) As prelayer and (d) Ga prelayer. (e) Various temperature light output against current characterization of InAs/GaAs QD laser monolithically grown on Ge substrate using Ga prelayer. Reproduced from reference [149].

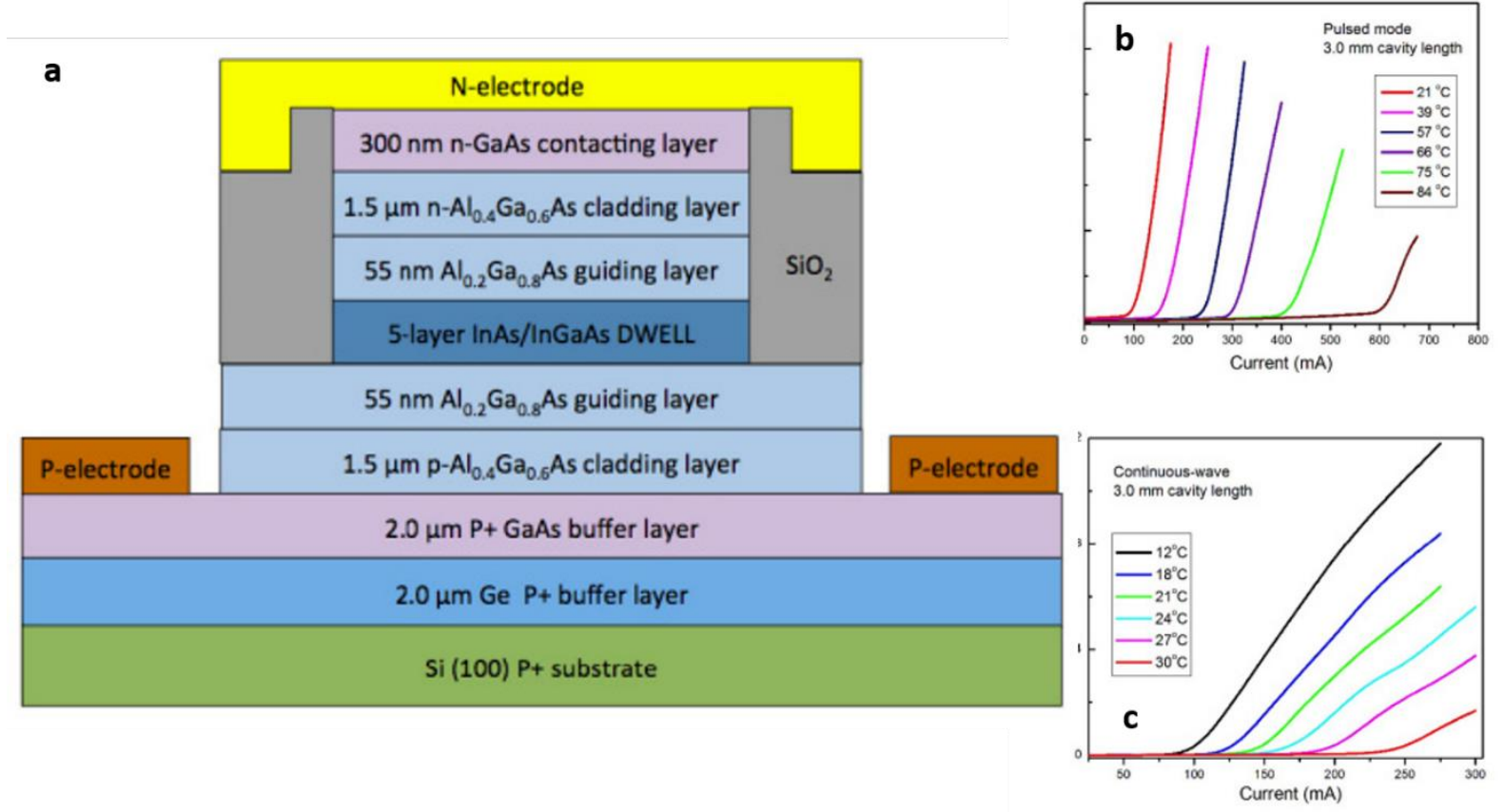

Fig. 10. InAs/GaAs QD laser monolithically grown on Ge/Si substrate. (a) Schematic diagram of 5 layers of InAs DWELL laser grown on Ge/Si substrate. Light output against injection current (L-I) at various heatsink temperature under (b) Pulsed mode and (c) CW mode. Reproduce from reference [150].

As the growth of $\mathrm{Ge}$ on $\mathrm{Si}$ has become a well-established and mature technology [157-159], the realization of high-performance III-V QD lasers grown on a Ge/Si substrate was demonstrated soon after the realization of III-V QD lasers on Ge substrates. To grow GaAs on a Ge/Si substrate, an off-cut substrate has been widely used to prevent APD formation. For instance, Lee et al. reported an InAs/GaAs QD lasers grown on (100)-oriented Si substrates with a $6^{\circ}$ offcut towards the [111] planes with a $2 \mu \mathrm{m}$-thick Ge buffer layer, as shown in Fig. 10(a) [150]. The Ga pre-layer technique was used to achieve a single-domain GaAs buffer. The laser structure consisted of a 5 layers dot-in-well (DWELL) active region within $1.5 \mu \mathrm{m} \mathrm{Al}{ }_{0.4} \mathrm{Ga}_{0.6}$ As upper and lower cladding layers. The measured $\mathrm{J}_{\text {th }}$ was 63.4 
$\mathrm{A} / \mathrm{cm}^{2}$ in pulsed mode and $163 \mathrm{~A} / \mathrm{cm}^{2}$ for room temperature $\mathrm{CW}$ operation with an output power $\sim 93$ $\mathrm{mW}$. Fig. 10(b) and (c) presents L-I measurements of the laser with maximum operating temperature of $84{ }^{\circ} \mathrm{C}$ and $30{ }^{\circ} \mathrm{C}$ under pulsed and $\mathrm{CW}$ mode, respectively. Liu et al. from UCSB demonstrated InAs/GaAs QD lasers monolithically grown on a Ge/Si substrate in collaboration with IQE Inc. in 2014 $[152,153]$. A high-temperature performance was achieved with a maximum operation temperature of $119{ }^{\circ} \mathrm{C}$ (excited state lasing) [153]. The active region comprised 7 layers of InAs/GaAs QDs emitting at $1252 \mathrm{~nm}$ wavelength as shown in Fig. 11(a) and the detailed layer structure is schematically illustrated in the inset of Fig. 11(a). The L-I measurements at temperatures ranging from $20{ }^{\circ} \mathrm{C}$ to $110{ }^{\circ} \mathrm{C}$ are presented in Fig. $11(\mathrm{~b})$, with a $\mathrm{T}_{0}$ value of $43 \mathrm{~K}$ obtained from $20^{\circ} \mathrm{C}$ to $110^{\circ} \mathrm{C}$. The single-side-emission wall plug efficiency reached $18 \%$ at an injection current of $150 \mathrm{~mA}$, corresponding to a differential efficiency of $\sim 37 \%$. Furthermore, a lifetime study was presented in the following year, which included testing for more than 2,700 hours (Fig. 11(c)) [160, 161]. So far, this is the best result, considering temperature performance and lifetime measurement for a laser grown on a $\mathrm{Ge} / \mathrm{Si}$ substrate.
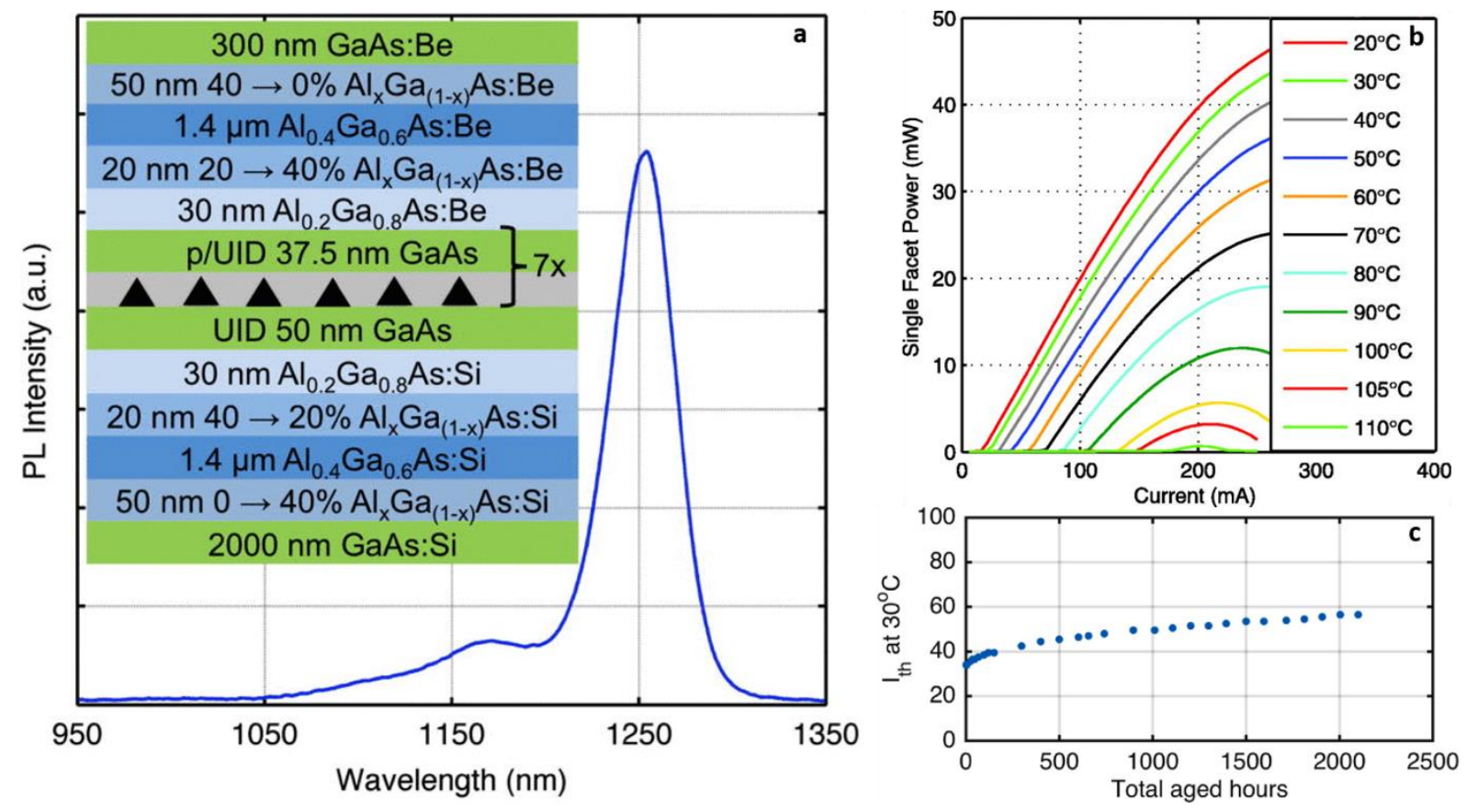

Fig. 11. InAs/GaAs QD laser grown on a Ge/Si substrate. (a) Room temperature photoluminescence spectrum of the 7 layers InAs/GaAs QD InAs quantum dots grown on Ge-on-Si substrates. Inset: layer structure of the InAs quantum dot lasers. (b) Temperature-dependent L-I measurement of the QD laser on $\mathrm{Ge} / \mathrm{Si}$ substrate. (c) Threshold current versus total aging time in hours for the InAs quantum dot lasers at $30{ }^{\circ} \mathrm{C}$. Reproduced from reference $[153,161]$.

\subsection{Heteroepitaxial growth of III-V lasers on Si substrates}

Recently, III-V lasers directly grown on Si substrates, without intermediate group IV buffer layers, have attracted considerable interest because of their potential to realize PICs on a Si platform directly compatible with Si CMOS techniques. However, the implementation of the III-V material directly grown on $\mathrm{Si}$ has been hampered by the high density of dislocations due to the lattice mismatch. In particular, the high density of threading dislocations (TDD) significantly reduces the performance of laser devices by creating electron scattering and a high ratio of non-radiative recombination centers which lower the external quantum efficiency [162-164]. This is mainly due to the formation of dangling bonds within dislocations acting as Auger recombination centers [165]. High-quality III-V materials grown on their native substrates (GaAs or InP) normally have a very low TDD $\left(<10^{5} \mathrm{~cm}^{-2}\right)$ [166]. On 
the other hand, GaAs directly grown on Si usually generates high TDD $\left(\sim 10^{10} \mathrm{~cm}^{-2}\right)$. Thus, a great deal of effort has been made to reduce the density of dislocations. For example, researchers from the University of Michigan demonstrated the first $1.1 \mu \mathrm{m}$ wavelength QD laser directly grown on $\mathrm{Si}$ substrate using a QD defect filter layer which can change the direction of threading dislocations [142144]. Furthermore, it was also reported that the introduction of multi-SLSs as defect filter layers reduced dislocation density [167-169]. The two directions of strain force along with the SLSs have potential to bend the threading dislocation propagation direction parallel to the SLSs, leading to the formation of misfit dislocations. As a result, the misfit dislocations can eliminate threading dislocations when they encounter each other with satisfying the condition that their Burgers vectors are perpendicular. Tang et al. also investigated the effect of different types of SLSs on the performance of QD lasers grown on $\mathrm{Si}$, and reported that the optimized InAs/GaAs SLS DFLs exhibit a lower threshold current density and higher operation temperature [146].

Besides the issue of high TDD, to integrate III-V on-chip light-emitting sources on a Si substrate and combine with other photonic components, a III-V laser grown on an exact (100) Si substrate has also been regarded as a critical issue to be solved. As we mentioned earlier, III-V materials directly grown on exact (100) Si and Ge substrates cause APDs. However, it was found that the APDs can be solved by using a special growth technique including GaAs or GaP intermediate buffer layer grown on Si exact (100) substrate by using MOCVD or MOVPE, or growing III-V materials on patterned Si substrate. The forthcoming laser devices based on these virtual substrate templates have been demonstrated very recently. 

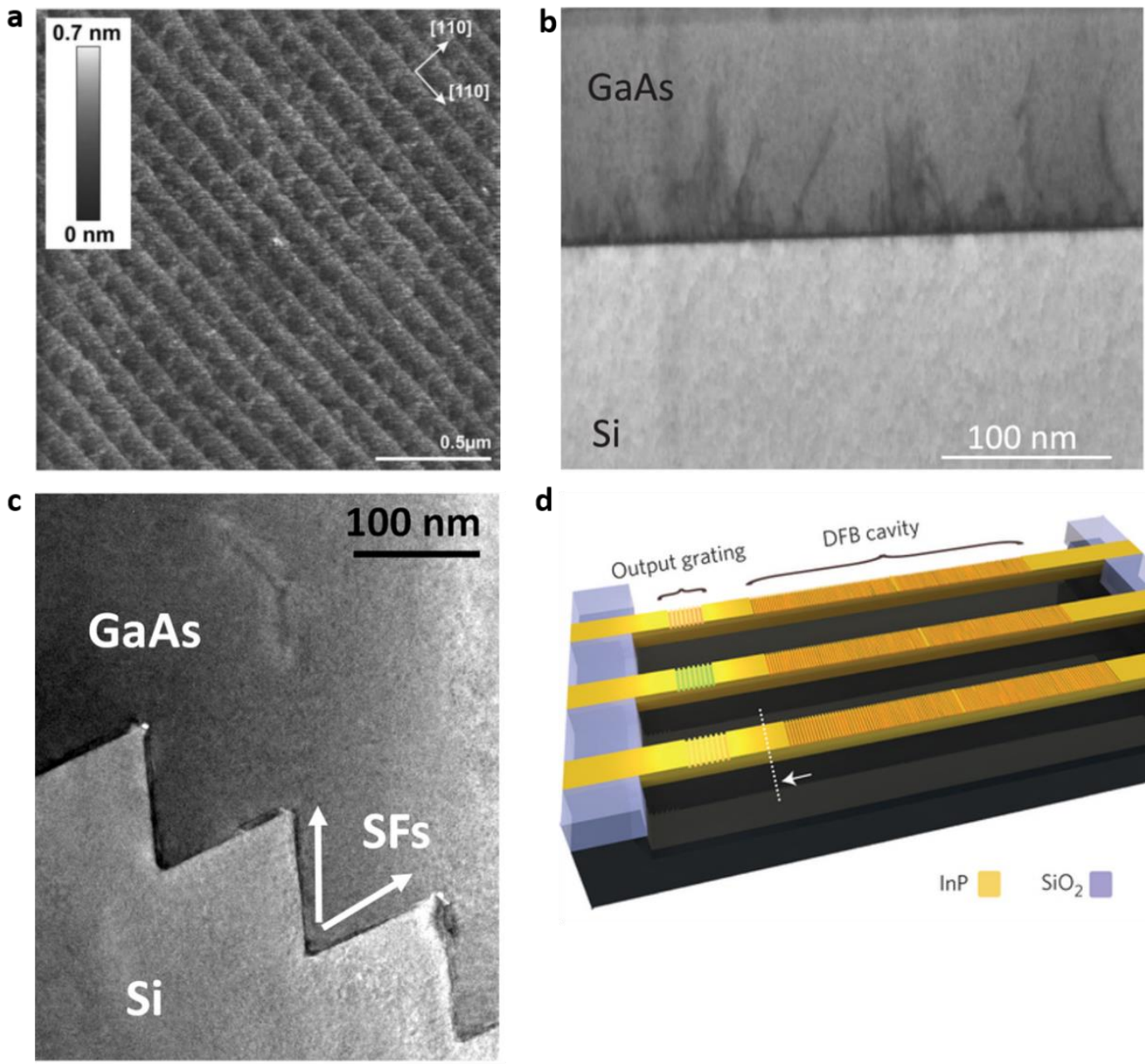

d

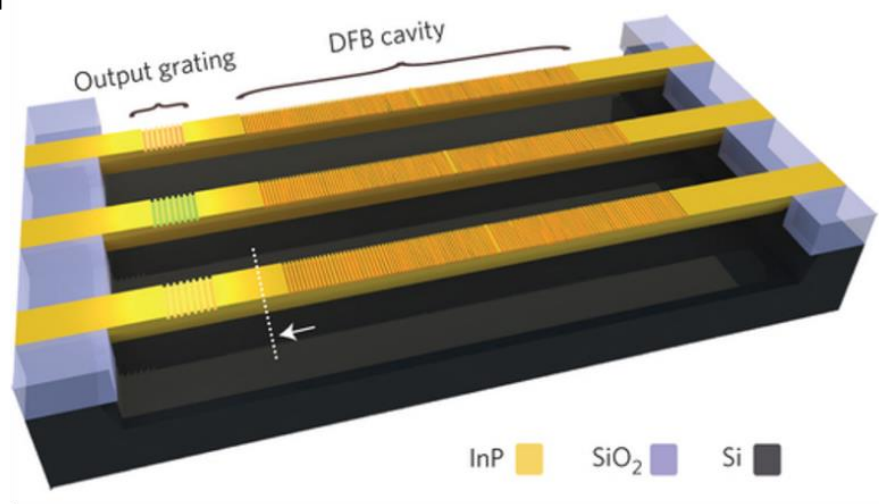

Fig. 12. III-V materials monolithically grown on on-axis $\mathrm{Si}$ substrate. (a) $\mathrm{GaP}$ on $\mathrm{Si}$ exact (100) substrate. (b) GaAs on a Si exact (100) substrate. (c) GaAs grown a Si V-grooved substrate. (d) InP laser grown on a patterned Si substrate. Reproduced from reference [170-173]

The studies of APD-free GaP grown on exact (100) Si substrates were developed by researchers from Philipps University Marburg, by using MOVPE and soon it was commercialized [170, 174, 175]. As shown in Fig. 12(a), a single-domain GaP layer was grown on a (001) exact orientated Si substrate with a very small offcut angle $\left(0.12^{\circ}\right)$ towards [1 10$]$, and the hydrogen annealing inside the MOVPE chamber was performed, which helps to destroy the $\pi$-bonding of $\mathrm{Si}$ atoms and reform as a double atomic step. Liu et al. successfully demonstrated an InAs/GaAs QD laser on this GaP/Si platform with CW operation and high operating temperature [176]. Very recently, Jung et al. from UCSB reported a significant improvement on a QD laser grown on GaP/Si substrate by using an InGaAs DFL, producing an extrapolated mean-time-to-failure of more than a million hours' lifetime and very low injection current of $6.2 \mathrm{~mA}[177,178]$. In addition to the GaP/Si platform, researchers from CEA-Leti (Grenoble, France) also developed the technique for APD-free GaAs epi-layers grown on 12-inch exact (100) Si substrates in 2016 [171]. After growth of 140-nm-thick GaAs, it was shown that APDs were eliminated due to the double step formation on Si substrates. Fig. 12(b) presents a cross-section TEM image of APD-free GaAs grown on on-axis (100) Si substrate. Researchers from UCL and CEA-Leti also demonstrated 1.3- $\mu \mathrm{m}$ QD lasers on exact (100) GaAs/Si substrate [179]. The $\mathrm{J}_{\text {th }}$ of the laser was measured to be $425 \mathrm{~A} / \mathrm{cm}^{2}$ at room temperature with the maximum operation temperature of $36{ }^{\circ} \mathrm{C}$ under $\mathrm{CW}$ mode. 
Besides using an intermediate buffer on the exact (100) Si substrate, growth on a patterned Si substrate has been also investigated. For instance, Li et al. reported APD-free GaAs thin film growth on a Vgrooved exact (001) Si substrate [167]. The V-grooved Si with a $60^{\circ}$ angle can confine the defects including APDs and stack faults within very low thickness of III-V materials. Fig. 12(c) shows an APDfree GaAs layer grown on a Si substrate, indicating the stacking faults lying on the V-grooved Si (111) plane. Wan et al. reported a room temperature study of InAs/GaAs QDs grown on a V-grooved Si substrate using MOCVD in 2015 [180]. QD microdisk lasers using this template were then demonstrated, which will be further discussed in the next section. In 2017, an electrical pumped CW FP QD ridge laser was demonstrated by Norman et al. [181].

Researchers from IMEC and University of Ghent reported an InP DFB array laser grown on patterned Si substrate in 2015, as shown in Fig. 12(d) [173, 182-184]. To fabricate the V-grooved Si substrate composed of two flat $\langle 111\rangle$ planes at the bottom of the trench, a $500 \mathrm{~nm}$-thick $\mathrm{SiO}_{2}$ ridge was selectively etched by using tetramethylammonium hydroxide (TMAH) solution. Then, the APD-free and highquality crystalline InP DFB lasers were grown within the trench by using MOVPE. The laser operation driven by an optical pumping source produced a threshold power of $22 \pm 2.1 \mathrm{~mW}$ with optical mode centered at $930.5 \mathrm{~nm}$, and ultimately reached more than $20 \mathrm{~dB}$ above the background. A further study on a single-mode InGaAs QW nano ridge laser was demonstrated very recently [185].

\subsection{High-performance F-P QD lasers}

A highly reliable QD laser directly grown on a Si substrate is essential for monolithic integration of a III-V on-chip light-emitting source on Si. In 2016, a $1.3 \mu \mathrm{m}$ QD laser directly grown on a Si substrate with long life time operating under continuous-wave mode was reported by Chen et al., based on their pervious extensive work [145, 147, 151, 167-169, 186-189]. This result indicates that the QD laser on $\mathrm{Si}$ is one step closer to realization in industrial use. To fabricate highly stable CW QD lasers grown on $\mathrm{Si}$, a fully relaxed $1 \mu \mathrm{m}$-thick GaAs buffer layer was grown on a $6 \mathrm{~nm}$-thick AlAs nucleation layer on the Si substrate. The GaAs buffer layer has been divided into three parts: the first $30 \mathrm{~nm}$-thick GaAs was grown at very low temperature of $350{ }^{\circ} \mathrm{C}$ at $0.1 \mathrm{ML} / \mathrm{s}$ to reduce the strain between the GaAs and $\mathrm{Si}$, while the second GaAs layer of $170 \mathrm{~nm}$ thickness was grown at middle temperature of $450{ }^{\circ} \mathrm{C}$ with $0.6 \mathrm{ML} / \mathrm{s}$ growth rate, which helps to release the strain and hence keeps the surface smooth at the same time. The remaining 800-nm-thick GaAs was grown at high temperature of $590{ }^{\circ} \mathrm{C}$ to fully relax the strain. In addition, the introduction of 5 sets of InGaAs/GaAs SLSs DFLs successfully reduced the TDD to the level of $10^{5} \mathrm{~cm}^{-2} .5$ layers of InAs/GaAs DWELL were embedded within $1.5 \mu \mathrm{m}$-thick $\mathrm{Al}_{0.4} \mathrm{Ga}_{0.6} \mathrm{As} \mathrm{n}$-type and p-type cladding layers. The fabricated laser has top-top contact configuration as can be seen in Fig. 13(a). Fig. 13(b) and (c) presents the SEM images of fabricated broad-area laser (cavity length of $3 \mathrm{~mm}$ with $50 \mu \mathrm{m}$ width stripes) with a mirror-like as-cleaved facet. An extremely low $\mathrm{J}_{\mathrm{th}}$ of $62.5 \mathrm{~A} / \mathrm{cm}^{2}$ was obtained at room temperature, together with a maximum operating temperature of $75^{\circ} \mathrm{C}$ under $\mathrm{CW}$ mode as shown in Fig. 13(d). The maximum operating temperature reached $120^{\circ} \mathrm{C}$ under pulsed operation. The measured output power from both facets was as high as $105 \mathrm{~mW}$ at an injection current density of $650 \mathrm{Acm}^{-2}$. As shown in Fig. 13(e), a 3100 hour ageing test was performed, from which an extrapolated lifetime (defined by a doubling of the threshold) of over 100,158 hours was calculated by fitting the threshold with a sublinear model. 

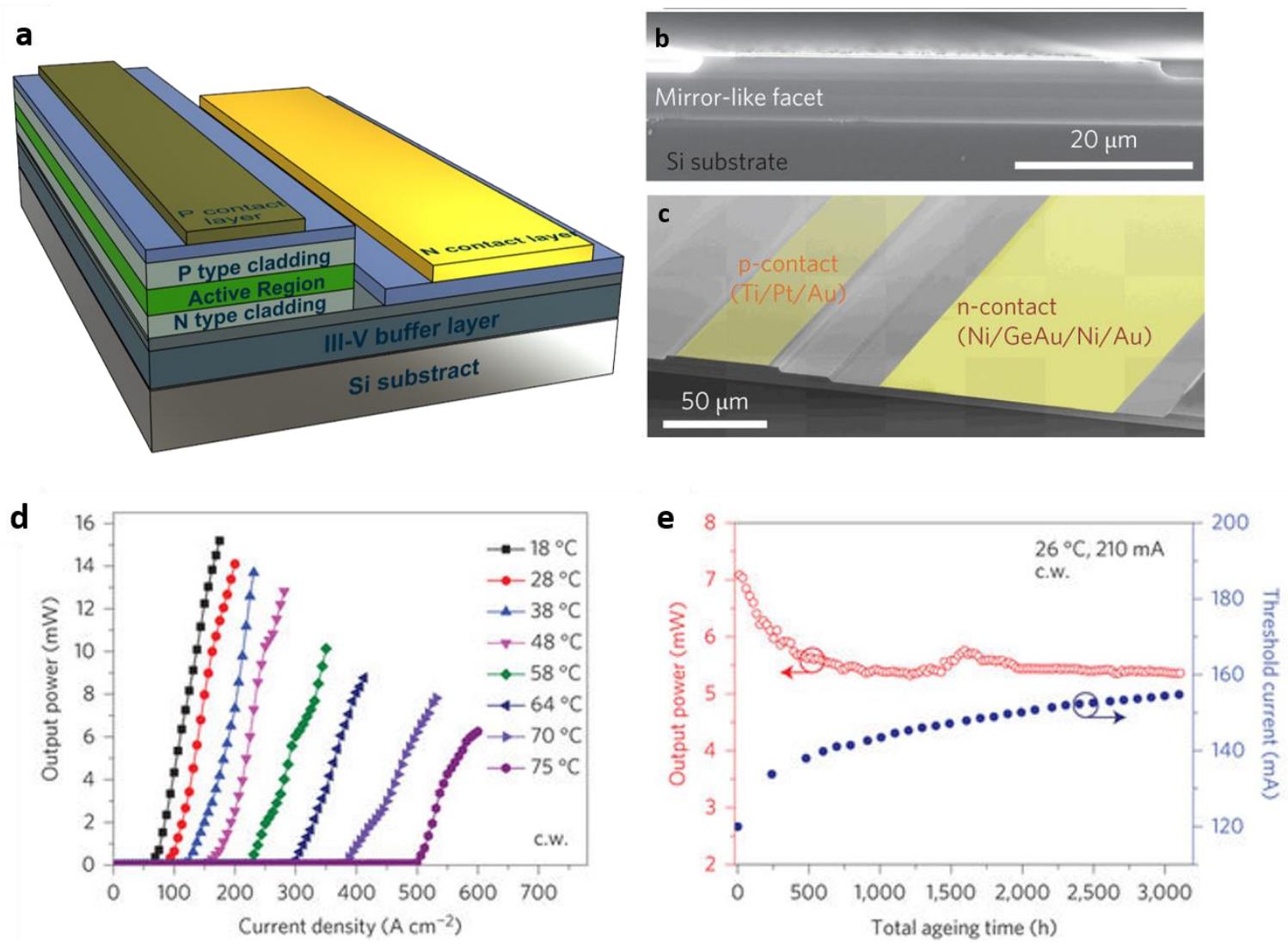

Fig. 13. InAs/GaAs QD laser directly grown on Si substrate. (a) Schematic diagram of laser structure. Scanning electron microscope images of (b) mirror-like facet without polishing, and (c) overview of the complete laser. (d) Temperature dependent L-I measurements. (e) Aging data of the laser measured under $26^{\circ} \mathrm{C}$ and $210 \mathrm{~mA}$ injection current with CW mode. Reproduced from reference [147].

\subsection{Microdisk laser grown on Si substrate}

Microdisk and microring lasers grown on Si substrates have several unique advantages such as CMOScompatibility, low power consumption, high-quality factor of the microcavity, and dense integration for optical transmitters due to the small laser footprint [190]. In this respect, a significant advance has been made in the realization of III-V/Si micro lasers. For example, optically pumped 1.1 and $1.3 \mu \mathrm{m}$ InAs/GaAs QD microdisk lasers grown on V-grooved Si (100) substrate were demonstrated by Wan and Li et al. from Hong Kong University of Science and Technology (HKUST) collaborating with UCSB in 2016 [191-193]. Furthermore, an electrically pumped InAs/GaAs QD laser grown on a Si substrate was realized by Kryzhanovskaya et al. from St. Petersburg Academic University collaborating with UCL [194]. As shown in Fig.14(a), the active region embedded within $1.5 \mu \mathrm{m}$ of $\mathrm{Al}_{0.4} \mathrm{Ga}_{0.6} \mathrm{As}$ nand p-type cladding layers consisted of 7 layers of DWELL structure, and the InGaAs/GaAs SLSs DFLs were grown on a $\mathrm{Si}$ (001) substrate with $4^{\circ}$ offcut to the [011] plane by MBE system. In Fig. 14(b), it was observed that the fabricated lasers with diameter of 14 and $30 \mu \mathrm{m}$ emitted around wavelength 1325 and $1355 \mathrm{~nm}$ at an injection current of 7.6 and $6.6 \mathrm{~mA}$, respectively, at room temperature without any external cooling and heatsink. 

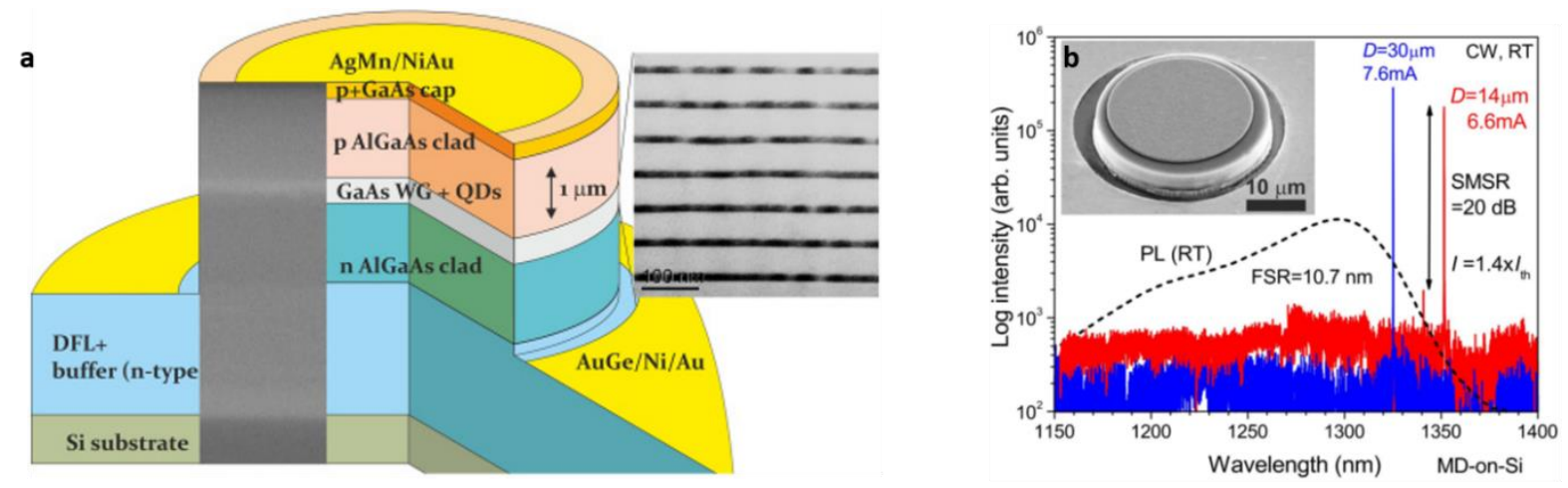

Fig. 14. InAs/GaAs QD microdisk laser monolithically grown on Si substrate. (a) Schematic diagram of the laser structure, left inset: cross-section SEM image of the grown epilayers; right inset: crosssection TEM image of InAs/GaAs QD region. (b) Lasing spectra of microdisk lasers with dimeter 30 and $14 \mu \mathrm{m}$ at room temperature. Inset: SEM image of the fabricated microdisk laser with dimeter 30 $\mu \mathrm{m}$. Reproduced from reference [194]

In the same year, 2017, researchers from HKUST and UCSB also developed electrically pumped QD micro-ring lasers grown on $\mathrm{GaP} / \mathrm{Si}$ (100) on-axis substrates and v-grooved $\mathrm{Si}$ substrates emitting in the O-band wavelength region with low $\mathrm{J}_{\text {th }}$, separately [195]. Moreover, $1.55 \mu \mathrm{m}$ wavelength microdisk lasers were demonstrated by the researchers from HKUST and Harvard University [196]. To achieve $1.55 \mu \mathrm{m}$ wavelength QDs, a strain relaxed InP buffer layer was grown on the top of GaAs buffer layer based on $\mathrm{Si}(001)$ substrate with slight unintentional miscuts ranging from $0.3^{\circ}$ to $0.8^{\circ}$. In addition, QD dislocation filters were also employed in this structure, reducing the TDD to $3.2 \times 10^{8} \mathrm{~cm}^{-2}$ (measured on the top layer of InP). A 7 layers InAs/InAlGaAs QD active region with dot density $4 \times 10^{10} \mathrm{~cm}^{-2}$ was inserted between two $70 \mathrm{~nm}$-thick $\mathrm{In}_{0.51} \mathrm{Al}_{0.49} \mathrm{As}$ cladding layers. Fig. 15(a) shows a schematic diagram of the fabricated microdisk laser with a diameter of $4 \mu \mathrm{m}$ and an etched InP pillar. A smooth sidewall was confirmed in Fig. 15(b), which significantly reduces the scattering loss. A telecommunication wavelength $(1578 \mathrm{~nm})$ lasing peak appeared under $\mathrm{CW}$ optical pump at temperature $4.5 \mathrm{~K}$ with an effective threshold power of $1.6 \mathrm{~mW}$, as shown in Fig. 15(c). Further improvements on the maximum operating temperature have been reported by Zhu $(173 \mathrm{~K})$ and Shi $\left(60^{\circ} \mathrm{C}\right)$ et al. $[197,198]$. 


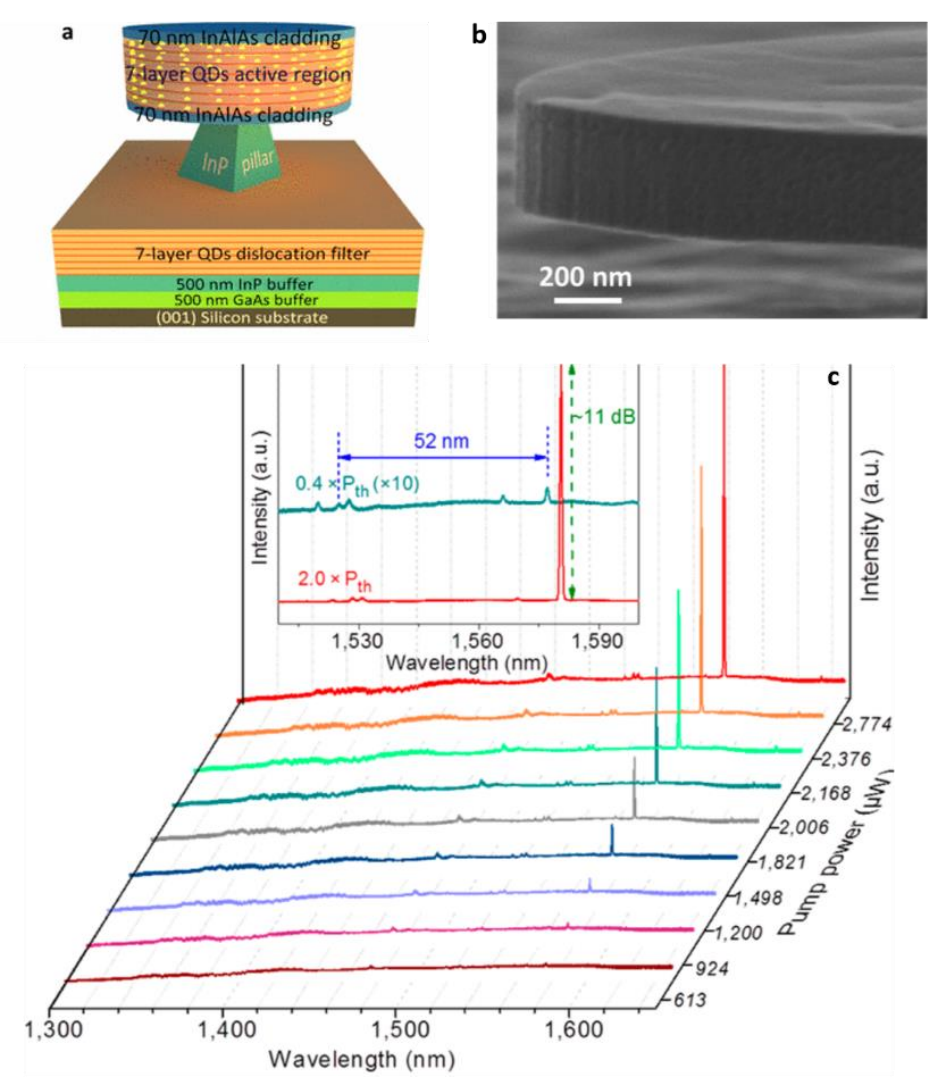

Fig. 15. $1.55 \mu \mathrm{m}$ wavelength $\mathrm{InAs} / \mathrm{InAlGaAs}$ QD microdisk laser grown on a Si substrate. (a) Schematic diagram of the fabricated laser structure. (b) SEM image of the side wall of microdisk laser. (c) Lasing spectra with different optical pump power; inset: high-resolution spectrum. Reproduced with permission [196]. Copyright (2018) American Chemical Society

\subsection{Summary of recent telecommunication wavelength QD lasers}

A summary of recent QD lasers directly grown on $\mathrm{Si}$ substrates without a Ge intermediate layer is presented in Table 1. Edge-emitting QD lasers demonstrated by UCL and UCSB have shown performance comparable to III-V lasers grown on native substrates. Currently, the best results for QD lasers grown on offcut and non-offcut Si substrates have reached the requirement of long lifetime, high operating temperature and high operating powers. It has also been proved that the performance of the laser devices grown on highly lattice mismatched Si substrate remains stable without significant degradation. Also, microdisk and microring $1.5 \mu \mathrm{m}$ wavelength QD lasers showed comparable performance on temperature and threshold properties, compared with $1.3 \mu \mathrm{m}$ wavelength QD lasers, due to the successful demonstration of InP buffer grown on Si substrate. 


\begin{tabular}{|c|c|c|c|c|c|c|c|}
\hline Institute & $\begin{array}{l}\text { Laser } \\
\text { Type }\end{array}$ & $\begin{array}{l}\lambda \\
(\mu \mathrm{m})\end{array}$ & $\mathrm{J}_{\mathrm{th}} / \mathrm{P}_{\mathrm{th}}$ & $\mathrm{T}_{\max }$ & Substrate & $\begin{array}{l}\text { Buffer } \\
\text { Layer }\end{array}$ & Ref \\
\hline UCL & $\begin{array}{l}\text { Edge- } \\
\text { emitting }\end{array}$ & 1.3 & $\begin{array}{l}\text { 62.5 } \mathrm{Acm}^{-2} \text { at } \\
\text { RT }\end{array}$ & $\begin{array}{l}120{ }^{\circ} \mathrm{C} \\
\text { (pulsed)/ } \\
75^{\circ} \mathrm{C}(\mathrm{CW})\end{array}$ & $\mathrm{Si} 4^{\circ}$ offcut & GaAs & [147] \\
\hline $\begin{array}{l}\text { UCSB + } \\
\text { University of } \\
\text { Illinois }\end{array}$ & $\begin{array}{l}\text { Edge- } \\
\text { emitting }\end{array}$ & 1.3 & $\begin{array}{lll}860 & \mathrm{Acm}^{-2} & \text { at } \\
\text { RT } & & \end{array}$ & $90^{\circ} \mathrm{C}(\mathrm{CW})$ & $\begin{array}{l}\text { Si on-axis } \\
(100)\end{array}$ & $\begin{array}{l}\text { GaAs/ } \\
\mathrm{GaP}\end{array}$ & [176] \\
\hline $\begin{array}{l}\text { UCSB } \\
\text { HKUST }\end{array}$ & $\begin{array}{l}\text { Edge- } \\
\text { emitting }\end{array}$ & 1.3 & $\begin{array}{l}500 \quad \mathrm{Acm}^{-2} \text { at } \\
\text { RT }\end{array}$ & $80^{\circ} \mathrm{C}(\mathrm{CW})$ & $\begin{array}{l}\text { V-grooved } \\
\mathrm{Si}\end{array}$ & GaAs & [181] \\
\hline $\begin{array}{l}\text { UCL + CEA } \\
\text { Leti }\end{array}$ & $\begin{array}{l}\text { Edge- } \\
\text { emitting }\end{array}$ & 1.3 & 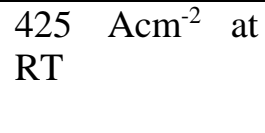 & $\begin{array}{l}102^{\circ} \mathrm{C} \\
(\mathrm{Pulse}) / 36 \\
{ }^{\circ} \mathrm{C}(\mathrm{CW})\end{array}$ & $\begin{array}{l}\mathrm{Si} \text { on-axis } \\
(100)\end{array}$ & GaAs & [179] \\
\hline UCSB & $\begin{array}{l}\text { Edge- } \\
\text { emitting }\end{array}$ & 1.3 & $\begin{array}{l}229.8 \mathrm{Acm}^{-2} \text { at } \\
\text { RT }\end{array}$ & $85^{\circ} \mathrm{C}(\mathrm{CW})$ & $\begin{array}{l}\text { Si on-axis } \\
(100)\end{array}$ & $\begin{array}{l}\text { GaAs/ } \\
\mathrm{GaP}\end{array}$ & [177] \\
\hline $\begin{array}{l}\text { HKUST } \quad+ \\
\text { UCSB }\end{array}$ & Microdisk & 1.3 & $\begin{array}{ll}250 & \mu \mathrm{W} \\
\text { (average) }\end{array}$ & RT & $\begin{array}{l}\text { V-grooved } \\
\mathrm{Si}\end{array}$ & GaAs & [192] \\
\hline $\begin{array}{l}\text { St. } \\
\text { Petersburg + } \\
\text { UCL }\end{array}$ & Microdisk & 1.3 & $600 \mathrm{Acm}^{-2}$ & RT (CW) & $\mathrm{Si} 4^{\circ}$ offcut & GaAs & [194] \\
\hline $\begin{array}{l}\text { UCSB+HKU } \\
\text { ST }\end{array}$ & Microring & 1.3 & $\begin{array}{l}875 \mathrm{Acm}^{-2} \\
\text { (average) }\end{array}$ & $60^{\circ} \mathrm{C}(\mathrm{CW})$ & $\begin{array}{l}\text { V-grooved } \\
\mathrm{Si}\end{array}$ & GaAs & [195] \\
\hline $\begin{array}{l}\text { UCSB+HKU } \\
\text { ST }\end{array}$ & Microring & 1.3 & $\begin{array}{l}675 \mathrm{Acm}^{-2} \\
\text { (average) }\end{array}$ & $80^{\circ} \mathrm{C}(\mathrm{CW})$ & $\begin{array}{l}\mathrm{Si} \text { on-axis } \\
(100)\end{array}$ & $\begin{array}{l}\text { GaAs/ } \\
\mathrm{GaP}\end{array}$ & [195] \\
\hline $\begin{array}{l}\text { HKUST }+ \\
\text { Harvard }\end{array}$ & Microdisk & 1.55 & $\begin{array}{ll}2.73 & \mu \mathrm{W} \\
\text { (pulsed) }\end{array}$ & $60^{\circ} \mathrm{C}$ & $\begin{array}{l}\mathrm{Si} \quad 0.3^{\circ} \text { to } \\
0.8^{\circ} \text { offcut }\end{array}$ & $\begin{array}{l}\mathrm{InP} / \mathrm{Ga} \\
\mathrm{As}\end{array}$ & [198] \\
\hline
\end{tabular}

Table 1. Summary of recent results of telecommunication wavelength InAs QD lasers monolithically grown on Si substrate with different methods.

\section{Discussion and conclusion}

We have reviewed recent progress on III-V lasers heterogeneously and monolithically integrated on the $\mathrm{Si}$ platform. Detailed reviews of recent InP-based DFB, mode-locked, circular microcavity and PhC nanocavity lasers bonding on $\mathrm{Si}$ have been presented. As a relatively mature technology, heterogeneous integration has successfully built light-emitting sources on Si substrates and integrated these with other photonic components owing to years of effort for combining the advantages of both III-V and $\mathrm{Si}$ technologies. With the realization of the Intel $100 \mathrm{G}$ optical transceiver, obviously, higher speed and bandwidth will be the next goals for Si photonics. Also back-end technology has been widely used in the Si-based PIC and OEIC integrated with CMOS, which facilitates the bonding of the III-V dies on $\mathrm{Si}$ or SOI platform. This technique requires to avoid the contaminations into III-V layers and obtain maximum $400^{\circ} \mathrm{C}$ thermal budget to circumvent the damage of electronic components [199]. However, we could find some degradation of III-V/Si hybrid laser performance, compared with the native III-V substrate. Moreover, heterogeneous integration does require a more complicated fabrication process than monolithic integration. In particular, the realization of a high-density of laser devices integrated on Si chips is a major challenge.

The previous major challenge of III-V lasers monolithically integrated on Si platform has been the impaired device performance due to the materials dissimilarity. Recently, III-V QD lasers monolithically grown on Si substrates have demonstrated very promising results with a long lifetime, high output power, and low threshold current densities, which are significantly better than those of Si- 
based QW lasers. Moreover, the single-device performance of monolithically integrated lasers has exceeded the performance of the heterogeneously integrated device. Monolithic integration also benefits from smaller parasitic capacitances and lower packaging cost [200]. In order to realize the application leveraging the monolithic integration of QD lasers on SOI platform, however, the optical coupling to waveguides must be resolved. Due to the thick buffer layer used in QD lasers grown on the $\mathrm{Si}$ offcut, on-axis and V-grooved platform, the strategies for coupling light into Si-based waveguide will be different from those used in heterogeneous integration. III-V lasers selectively grown with-in the Si trench may solve the problem, but this technique is likely to be hampered by the imperfect crystal quality. III-V crystals may form as polycrystalline during the selective growth [201]. More effort is clearly required in this area in the very near future. Nevertheless, Si-based III-V QD lasers provide a new platform for III-V/Si integration and are the most promising solution for achieving reliable, power efficient, high-density integration of laser diodes on silicon chips.

\section{Acknowledgments}

This work was supported by UK Engineering and Physical Sciences Research Council (EPSRC) [grant numbers EP/J012904/1, EP/P000886/1, EP/P006973/1] and EPSRC National Epitaxy Facility; S.C. thanks for the Royal Academy of Engineering for funding his Research Fellowship.

[1] H. Izuo, Optoelectronic Devices and Material Technologies for Photo-Electronic Integrated Systems, Japanese Journal of Applied Physics 32 (1993) 266.

[2] B. Jalali, S. Fathpour, Silicon Photonics, Journal of Lightwave Technology 24 (2006) 4600-4615.

[3] D.A.B. Miller, Rationale and challenges for optical interconnects to electronic chips, Proceedings of the IEEE 88 (2000) 728-749.

[4] R. Soref, Mid-infrared photonics in silicon and germanium, Nature Photonics 4 (2010) 495.

[5] R. Soref, The Past, Present, and Future of Silicon Photonics, IEEE Journal of Selected Topics in Quantum Electronics 12 (2006) 1678-1687.

[6] I. Kaminow, Optical Integrated Circuits: A Personal Perspective, Journal of Lightwave Technology 26 (2008) 994-1004.

[7] J.A. Kash, A.F. Benner, F.E. Doany, D.M. Kuchta, B.G. Lee, P.K. Pepeljugoski, L. Schares, C.L. Schow, M. Taubenblatt, in: 2011 Optical Fiber Communication Conference and Exposition and the National Fiber Optic Engineers Conference, (2011).

[8] M. Haurylau, G. Chen, H. Chen, J. Zhang, N.A. Nelson, D.H. Albonesi, E.G. Friedman, P.M. Fauchet, On-Chip Optical Interconnect Roadmap: Challenges and Critical Directions, IEEE Journal of Selected Topics in Quantum Electronics 12 (2006) 1699-1705.

[9] R.A. Soref, Silicon-based optoelectronics, Proceedings of the IEEE 81 (1993) 1687-1706.

[10] R. Nagarajan, M. Kato, J. Pleumeekers, P. Evans, S. Corzine, S. Hurtt, A. Dentai, S. Murthy, M. Missey, R. Muthiah, R.A. Salvatore, C. Joyner, R. Schneider, M. Ziari, F. Kish, D. Welch, InP Photonic Integrated Circuits, IEEE Journal of Selected Topics in Quantum Electronics 16 (2010) 1113-1125.

[11] P. Evans, M. Fisher, R. Malendevich, A. James, P. Studenkov, G. Goldfarb, T. Vallaitis, M. Kato, P. Samra, S. Corzine, E. Strzelecka, R. Salvatore, F. Sedgwick, M. Kuntz, V. Lal, D. Lambert, A. Dentai, D. Pavinski, B. Behnia, J. Bostak, V. Dominic, A. Nilsson, B. Taylor, J. Rahn, S. Sanders, H. Sun, K.-T. Wu, J. Pleumeekers, R. Muthiah, M. Missey, R. Schneider, J. Stewart, M. Reffle, T. Butrie, R. Nagarajan, C. Joyner, M. Ziari, F. Kish, D. Welch, in: Optical Fiber Communication Conference/National Fiber Optic Engineers Conference 2011, (Optical Society of America, Los Angeles, California, 2011).

[12] D.F. Welch, F.A. Kish, S. Melle, R. Nagarajan, M. Kato, C.H. Joyner, J.L. Pleumeekers, R.P. Schneider, J. Back, A.G. Dentai, V.G. Dominic, P.W. Evans, M. Kauffman, D.J.H. Lambert, S.K. Hurtt, A. Mathur, M.L. Mitchell, M. Missey, S. Murthy, A.C. Nilsson, R.A. Salvatore, M.F.V. Leeuwen, J. Webjorn, M. Ziari, S.G. Grubb, D. Perkins, M. Reffle, D.G. Mehuys, Large-Scale InP Photonic Integrated Circuits: Enabling 
Efficient Scaling of Optical Transport Networks, IEEE Journal of Selected Topics in Quantum Electronics 13 (2007) 22-31.

[13] Y.A. Vlasov, Silicon CMOS-integrated nano-photonics for computer and data communications beyond 100G, IEEE Communications Magazine 50 (2012) s67-s72.

[14] D. Liang, J.E. Bowers, Photonic integration: Si or InP substrates?, Electronics Letters 45 (2009) 578581.

[15] M. Asghari, A.V. Krishnamoorthy, Energy-efficient communication, Nature Photonics 5 (2011) 268. [16] M.J.R. Heck, J.E. Bowers, Energy Efficient and Energy Proportional Optical Interconnects for MultiCore Processors: Driving the Need for On-Chip Sources, IEEE Journal of Selected Topics in Quantum Electronics 20 (2014) 332-343.

[17] D. Dai, J. Bauters, J.E. Bowers, Passive technologies for future large-scale photonic integrated circuits on silicon: polarization handling, light non-reciprocity and loss reduction, Light: Science \&Amp; Applications 1 (2012) e1.

[18] M. Streshinsky, R. Ding, Y. Liu, A. Novack, Y. Yang, Y. Ma, X. Tu, E.K.S. Chee, A.E.-J. Lim, P.G.-Q. Lo, T. Baehr-Jones, M. Hochberg, Low power $50 \mathrm{~Gb} / \mathrm{s}$ silicon traveling wave Mach-Zehnder modulator near 1300 nm, Optics Express 21 (2013) 30350-30357.

[19] X. Tu, T.-Y. Liow, J. Song, X. Luo, Q. Fang, M. Yu, G.-Q. Lo, 50-Gb/s silicon optical modulator with traveling-wave electrodes, Optics Express 21 (2013) 12776-12782.

[20] T. Yin, R. Cohen, M.M. Morse, G. Sarid, Y. Chetrit, D. Rubin, M.J. Paniccia, 31GHz Ge n-i-p waveguide photodetectors on Silicon-on-Insulator substrate, Optics Express 15 (2007) 13965-13971.

[21] L. Vivien, A. Polzer, D. Marris-Morini, J. Osmond, J.M. Hartmann, P. Crozat, E. Cassan, C. Kopp, H. Zimmermann, J.M. Fédéli, Zero-bias 40Gbit/s germanium waveguide photodetector on silicon, Optics Express 20 (2012) 1096-1101.

[22] J. Michel, J. Liu, L.C. Kimerling, High-performance Ge-on-Si photodetectors, Nature Photonics 4 (2010) 527.

[23] D. Liang, J.E. Bowers, Recent progress in lasers on silicon, Nat Photon 4 (2010) 511-517.

[24] J. Liu, X. Sun, D. Pan, X. Wang, L.C. Kimerling, T.L. Koch, J. Michel, Tensile-strained, n-type Ge as a gain medium for monolithic laser integration on Si, Optics Express 15 (2007) 11272-11277.

[25] J. Liu, X. Sun, R. Camacho-Aguilera, L.C. Kimerling, J. Michel, Ge-on-Si laser operating at room temperature, Optics Letters 35 (2010) 679-681.

[26] R.E. Camacho-Aguilera, Y. Cai, N. Patel, J.T. Bessette, M. Romagnoli, L.C. Kimerling, J. Michel, An electrically pumped germanium laser, Optics Express 20 (2012) 11316-11320.

[27] S. Wirths, R. Geiger, N. von den Driesch, G. Mussler, T. Stoica, S. Mantl, Z. Ikonic, M. Luysberg, S. Chiussi, J.M. Hartmann, H. Sigg, J. Faist, D. Buca, D. Grützmacher, Lasing in direct-bandgap GeSn alloy grown on Si, Nat Photon 9 (2015) 88-92.

[28] H. Rong, R. Jones, A. Liu, O. Cohen, D. Hak, A. Fang, M. Paniccia, A continuous-wave Raman silicon laser, Nature 433 (2005) 725-728.

[29] H. Rong, A. Liu, R. Jones, O. Cohen, D. Hak, R. Nicolaescu, A. Fang, M. Paniccia, An all-silicon Raman laser, Nature 433 (2005) 292-294.

[30] M. Aoki, M. Suzuki, H. Sano, T. Kawano, T. Ido, T. Taniwatari, K. Uomi, A. Takai, InGaAs/InGaAsP MQW Electroabsorption Modulator Integrated with a DFB Laser Fabricated by Band-Gap Energy Control Selective Area MOCVD, IEEE Journal of Quantum Electronics 29 (1993) 2088-2096.

[31] S. Banyoudeh, A. Abdollahinia, O. Eyal, F. Schnabel, V. Sichkovskyi, G. Eisenstein, J. Reithmaier, Temperature-Insensitive High-Speed Directly Modulated 1.55 $\mu \mathrm{m}$ Quantum Dot Lasers, IEEE Photonics Technology Letters 1135 (2016) 1-1.

[32] S. Banyoudeh, A. Abdollahinia, V. Sichkovskyi, J.P. Reithmaier, 1. $5 \mathrm{Mm}$ Quantum Dot Laser Material With High Temperature Stability of Threshold Current Density and External Differential Efficiency, 9767 (2016) 1-7.

[33] D. Gready, G. Eisenstein, V. Ivanov, C. Gilfert, F. Schnabel, A. Rippien, J.P. Reithmaier, C. Bornholdt, V. Ivanov, J.P. Reithmaier, High Speed $1.55 \mu \mathrm{m}$ InAs/InGaAlAs/InP Quantum Dot Lasers, IEEE Photonics Technology Letters 26 (2014) 11-13. 
[34] H.Y. Liu, I.R. Sellers, T.J. Badcock, D.J. Mowbray, M.S. Skolnick, K.M. Groom, M. Gutiérrez, M. Hopkinson, J.S. Ng, J.P.R. David, R. Beanland, Improved performance of $1.3 \mu \mathrm{m}$ multilayer InAs quantum-dot lasers using a high-growth-temperature GaAs spacer layer, Applied Physics Letters 85 (2004) 704-706.

[35] H.Y. Liu, D.T. Childs, T.J. Badcock, K.M. Groom, I.R. Sellers, M. Hopkinson, R.A. Hogg, D.J. Robbins, D.J. Mowbray, M.S. Skolnick, High-performance three-layer 1.3- $\mu \mathrm{m}$ InAs-GaAs quantum-dot lasers with very low continuous-wave room-temperature threshold currents, IEEE Photonics Technology Letters 17 (2005) 1139-1141.

[36] O.B. Shchekin, D.G. Deppe, $1.3 \mu \mathrm{m}$ InAs quantum dot laser with $\mathrm{To}=161 \mathrm{~K}$ from 0 to $80^{\circ} \mathrm{C}$, Applied Physics Letters 80 (2002) 3277-3279.

[37] D.L. Huffaker, G. Park, Z. Zou, O.B. Shchekin, D.G. Deppe, Continuous-wave low-threshold performance of 1.3-/spl mu/m InGaAs-GaAs quantum-dot lasers, IEEE Journal of Selected Topics in Quantum Electronics 6 (2000) 452-461.

[38] N. Kirstaedter, O.G. Schmidt, N.N. Ledentsov, D. Bimberg, V.M. Ustinov, A.Y. Egorov, A.E. Zhukov, M.V. Maximov, P.S. Kop'ev, Z.I. Alferov, Gain and differential gain of single layer InAs/GaAs quantum dot injection lasers, Applied Physics Letters 69 (1996) 1226-1228.

[39] D. Bimberg, N. Kirstaedter, N.N. Ledentsov, Z.I. Alferov, P.S. Kop'ev, V.M. Ustinov, InGaAs-GaAs quantum-dot lasers, IEEE Journal of Selected Topics in Quantum Electronics 3 (1997) 196-205.

[40] G. Liu, A. Stintz, H. Li, K.J. Malloy, L.F. Lester, Extremely low room-temperature threshold current density diode lasers using $\operatorname{InAs}$ dots in $\ln _{0.15} \mathrm{Ga}_{0.85}$ As quantum well, Electronics Letters 35 (1999) 1163 1165.

[41] D.L. Huffaker, G. Park, Z. Zou, O.B. Shchekin, D.G. Deppe, $1.3 \mu \mathrm{m}$ room-temperature GaAs-based quantum-dot laser, Applied Physics Letters 73 (1998) 2564-2566.

[42] H. Altug, D. Englund, J. Vučković, Ultrafast photonic crystal nanocavity laser, Nature Physics 2 (2006) 484.

[43] S. Strauf, K. Hennessy, M.T. Rakher, Y.S. Choi, A. Badolato, L.C. Andreani, E.L. Hu, P.M. Petroff, D. Bouwmeester, Self-Tuned Quantum Dot Gain in Photonic Crystal Lasers, Physical Review Letters 96 (2006) 127404.

[44] D.G. Deppe, H. Huang, O.B. Shchekin, Modulation characteristics of quantum-dot lasers: the influence of $p$-type doping and the electronic density of states on obtaining high speed, IEEE Journal of Quantum Electronics 38 (2002) 1587-1593.

[45] H.Y. Liu, M. Hopkinson, C.N. Harrison, M.J. Steer, R. Frith, I.R. Sellers, D.J. Mowbray, M.S. Skolnick, Optimizing the growth of $1.3 \mu \mathrm{m}$ InAs/InGaAs dots-in-a-well structure, Journal of Applied Physics 93 (2003) 2931-2936.

[46] L. Esaki, R. Tsu, Superlattice and Negative Differential Conductivity in Semiconductors, IBM Journal of Research and Development 14 (1970) 61-65.

[47] P. Bhattacharya, J. Singh, H. Yoon, Z. Xiangkun, A. Gutierrez-Aitken, L. Yeeloy, Tunneling injection lasers: a new class of lasers with reduced hot carrier effects, IEEE Journal of Quantum Electronics 32 (1996) 1620-1629.

[48] K. Masahiko, N. Shin'ichi, K. Takeshi, Y. Yoshiaki, O. Makoto, Room-Temperature Pulsed Operation of GaInNAs Laser Diodes with Excellent High-Temperature Performance, Japanese Journal of Applied Physics 35 (1996) 5711.

[49] T.J. Badcock, H.Y. Liu, K.M. Groom, C.Y. Jin, M. Gutiérrez, M. Hopkinson, D.J. Mowbray, M.S. Skolnick, in: Electronics Letters, (Institution of Engineering and Technology, 2006).

[50] H.Y. Liu, S.L. Liew, T. Badcock, D.J. Mowbray, M.S. Skolnick, S.K. Ray, T.L. Choi, K.M. Groom, B. Stevens, F. Hasbullah, C.Y. Jin, M. Hopkinson, R.A. Hogg, p-doped $1.3 \mu \mathrm{m}$ InAs / GaAs quantum-dot laser with a low threshold current density and high differential efficiency, Applied Physics Letters 89 (2006) 073113.

[51] C.Y. Jin, T.J. Badcock, H.Y. Liu, K.M. Groom, R.J. Royce, D.J. Mowbray, M. Hopkinson, Observation and Modeling of a Room-Temperature Negative Characteristic Temperature 1.3- $\mu \mathrm{m}$ p-Type Modulation-Doped Quantum-Dot Laser, IEEE Journal of Quantum Electronics 42 (2006) 1259-1265. 
[52] I.C. Sandall, P.M. Smowton, C.L. Walker, T. Badcock, D.J. Mowbray, H.Y. Liu, M. Hopkinson, The effect of $p$ doping in InAs quantum dot lasers, Applied Physics Letters 88 (2006) 111113.

[53] P.M. Smowton, I.C. Sandall, D.J. Mowbray, H.Y. Liu, M. Hopkinson, Temperature-Dependent Gain and Threshold in P-Doped Quantum Dot Lasers, IEEE Journal of Selected Topics in Quantum Electronics 13 (2007) 1261-1266.

[54] T.J. Badcock, R.J. Royce, D.J. Mowbray, M.S. Skolnick, H.Y. Liu, M. Hopkinson, K.M. Groom, Q. Jiang, Low threshold current density and negative characteristic temperature $1.3 \mu \mathrm{m} \operatorname{lnAs}$ selfassembled quantum dot lasers, Applied Physics Letters 90 (2007) 111102.

[55] O.B. Shchekin, J. Ahn, D.G. Deppe, in: Electronics Letters, (Institution of Engineering and Technology, 2002).

[56] M. Sugawara, N. Hatori, M. Ishida, H. Ebe, Y. Arakawa, T. Akiyama, K. Otsubo, T. Yamamoto, Y. Nakata, Recent progress in self-assembled quantum-dot optical devices for optical telecommunication: temperature-insensitive $10 \mathrm{Gbs}-1$ directly modulated lasers and $40 \mathrm{~Gb} \mathrm{~s}-1$ signal-regenerative amplifiers, Journal of Physics D: Applied Physics 38 (2005) 2126.

[57] B. Corbett, R. Loi, W. Zhou, D. Liu, Z. Ma, Transfer print techniques for heterogeneous integration of photonic components, Progress in Quantum Electronics 52 (2017) 1-17.

[58] Z. Wang, K. Van Gasse, V. Moskalenko, S. Latkowski, E. Bente, B. Kuyken, G. Roelkens, A III-V-onSi ultra-dense comb laser, Light Sci Appl. 6 (2017) e16260.

[59] Y.-H. Jhang, R. Mochida, K. Tanabe, K. Takemasa, M. Sugawara, S. Iwamoto, Y. Arakawa, Direct modulation of $1.3 \mu \mathrm{m}$ quantum dot lasers on silicon at $60^{\circ} \mathrm{C}$, Optics Express 24 (2016) 18428-18435.

[60] A.W. Fang, H. Park, O. Cohen, R. Jones, M.J. Paniccia, J.E. Bowers, Electrically pumped hybrid AlGalnAs-silicon evanescent laser, Optics Express 14 (2006) 9203-9210.

[61] G. Roelkens, L. Liu, D. Liang, R. Jones, A. Fang, B. Koch, J. Bowers, III-V/silicon photonics for onchip and intra-chip optical interconnects, Laser \& Photonics Reviews 4 (2010) 751-779.

[62] D. Liang, G. Roelkens, R. Baets, J. Bowers, Hybrid Integrated Platforms for Silicon Photonics, Materials 3 (2010) 1782.

[63] G. Roelkens, J. Van Campenhout, J. Brouckaert, D. Van Thourhout, R. Baets, P.R. Romeo, P. Regreny, A. Kazmierczak, C. Seassal, X. Letartre, G. Hollinger, J.M. Fedeli, L. Di Cioccio, C. LagaheBlanchard, III-V/Si photonics by die-to-wafer bonding, Materials Today 10 (2007) 36-43.

[64] D. Sparks, G. Queen, R. Weston, G. Woodward, M. Putty, L. Jordan, S. Zarabadi, K. Jayakar, Waferto-wafer bonding of nonplanarized MEMS surfaces using solder, Journal of Micromechanics and Microengineering 11 (2001) 630.

[65] Y.T. Cheng, L. Lin, K. Najafi, Localized silicon fusion and eutectic bonding for MEMS fabrication and packaging, Journal of Microelectromechanical Systems 9 (2000) 3-8.

[66] M.M. Maharbiz, M.B. Cohn, R.T. Howe, R. Horowitz, A.P. Pisano, in: Technical Digest. IEEE International MEMS 99 Conference. Twelfth IEEE International Conference on Micro Electro Mechanical Systems (Cat. No.99CH36291), (1999).

[67] K. Jongbaeg, C. Mu, L. Liwei, in: Technical Digest. MEMS 2002 IEEE International Conference. Fifteenth IEEE International Conference on Micro Electro Mechanical Systems (Cat. No.02CH37266), (2002).

[68] S.A. Audet, K.M. Edenfeld, in: Solid State Sensors and Actuators, 1997. TRANSDUCERS '97 Chicago., 1997 International Conference on, (1997).

[69] K. Schjølberg-Henriksen, S. Moe, M.M.V. Taklo, P. Storås, J.H. Ulvensøen, Low-temperature plasma activated bonding for a variable optical attenuator, Sensors and Actuators A: Physical 142 (2008) 413-420.

[70] D. Pasquariello, K. Hjort, Plasma-assisted InP-to-Si low temperature wafer bonding, IEEE Journal of Selected Topics in Quantum Electronics 8 (2002) 118-131.

[71] S. Keyvaninia, M. Muneeb, S. Stanković, P.J. Van Veldhoven, D. Van Thourhout, G. Roelkens, Ultrathin DVS-BCB adhesive bonding of III-V wafers, dies and multiple dies to a patterned silicon-oninsulator substrate, Optical Materials Express 3 (2013) 35-46. 
[72] Y.H. Lo, R. Bhat, D.M. Hwang, C. Chua, C.H. Lin, Semiconductor lasers on Si substrates using the technology of bonding by atomic rearrangement, Applied Physics Letters 62 (1993) 1038-1040.

[73] H. Wada, T. Kamijoh, Room-temperature CW operation of InGaAsP lasers on Si fabricated by wafer bonding, IEEE Photonics Technology Letters 8 (1996) 173-175.

[74] K. Mori, K. Tokutome, S. Sugou, Low-threshold pulsed operation of long-wavelength lasers on Si fabricated by direct bonding, Electronics Letters 31 (1995) 284-285.

[75] H. Park, A.W. Fang, S. Kodama, J.E. Bowers, Hybrid silicon evanescent laser fabricated with a silicon waveguide and III-V offset quantum wells, Optics Express 13 (2005) 9460-9464.

[76] A.W. Fang, E. Lively, Y.-H. Kuo, D. Liang, J.E. Bowers, A distributed feedback silicon evanescent laser, Optics Express 16 (2008) 4413-4419.

[77] A.W. Fang, B.R. Koch, R. Jones, E. Lively, D. Liang, Y.H. Kuo, J.E. Bowers, A Distributed Bragg Reflector Silicon Evanescent Laser, IEEE Photonics Technology Letters 20 (2008) 1667-1669.

[78] D. Liang, J.E. Bowers, Highly efficient vertical outgassing channels for low-temperature InP-tosilicon direct wafer bonding on the silicon-on-insulator substrate, Journal of Vacuum Science \& Technology B: Microelectronics and Nanometer Structures Processing, Measurement, and Phenomena 26 (2008) 1560-1568.

[79] H. Park, A.W. Fang, O. Cohen, R. Jones, M.J. Paniccia, J.E. Bowers, A Hybrid AlGalnAs\&ndash;Silicon Evanescent Amplifier, IEEE Photonics Technology Letters 19 (2007) 230-232.

[80] B.R. Koch, A.W. Fang, O. Cohen, J.E. Bowers, Mode-locked silicon evanescent lasers, Optics Express 15 (2007) 11225-11233.

[81] H.-H. Chang, A.W. Fang, M.N. Sysak, H. Park, R. Jones, O. Cohen, O. Raday, M.J. Paniccia, J.E. Bowers, 1310nm silicon evanescent laser, Optics Express 15 (2007) 11466-11471.

[82] A.W. Fang, P. Hyundai, R. Jones, O. Cohen, M.J. Paniccia, J.E. Bowers, A continuous-wave hybrid AlGalnAs-silicon evanescent laser, IEEE Photonics Technology Letters 18 (2006) 1143-1145.

[83] G.H. Duan, C. Jany, A.L. Liepvre, A. Accard, M. Lamponi, D. Make, P. Kaspar, G. Levaufre, N. Girard, F. Lelarge, J.M. Fedeli, A. Descos, B.B. Bakir, S. Messaoudene, D. Bordel, S. Menezo, G.d. Valicourt, S. Keyvaninia, G. Roelkens, D.V. Thourhout, D.J. Thomson, F.Y. Gardes, G.T. Reed, Hybrid III--V on Silicon Lasers for Photonic Integrated Circuits on Silicon, IEEE Journal of Selected Topics in Quantum Electronics 20 (2014) 158-170.

[84] S. Keyvaninia, S. Verstuyft, L. Van Landschoot, F. Lelarge, G.H. Duan, S. Messaoudene, J.M. Fedeli, T. De Vries, B. Smalbrugge, E.J. Geluk, J. Bolk, M. Smit, G. Morthier, D. Van Thourhout, G. Roelkens, Heterogeneously integrated III-V/silicon distributed feedback lasers, Optics Letters 38 (2013) 54345437.

[85] W. Bogaerts, P. De Heyn, T. Van Vaerenbergh, K. De Vos, S. Kumar Selvaraja, T. Claes, P. Dumon, P. Bienstman, D. Van Thourhout, R. Baets, Silicon microring resonators, Laser \& Photonics Reviews 6 (2012) 47-73.

[86] S. Stankovic, R. Jones, M.N. Sysak, J.M. Heck, G. Roelkens, D.V. Thourhout, Hybrid III-V/Si Distributed-Feedback Laser Based on Adhesive Bonding, IEEE Photonics Technology Letters 24 (2012) 2155-2158.

[87] J. Van Campenhout, P. Rojo-Romeo, P. Regreny, C. Seassal, D. Van Thourhout, S. Verstuyft, L. Di Cioccio, J.M. Fedeli, C. Lagahe, R. Baets, Electrically pumped InP-based microdisk lasers integrated with a nanophotonic silicon-on-insulator waveguide circuit, Optics Express 15 (2007) 6744-6749.

[88] G. Roelkens, D.V. Thourhout, R. Baets, R. Nötzel, M. Smit, Laser emission and photodetection in an InP/InGaAsP layer integrated on and coupled to a Silicon-on-Insulator waveguide circuit, Optics Express 14 (2006) 8154-8159.

[89] S. Stankovic, R. Jones, M.N. Sysak, J.M. Heck, G. Roelkens, D.V. Thourhout, 1310-nm Hybrid IIIV/Si Fabry-Perot Laser Based on Adhesive Bonding, IEEE Photonics Technology Letters 23 (2011) 17811783.

[90] M. Lamponi, S. Keyvaninia, C. Jany, F. Poingt, F. Lelarge, G.d. Valicourt, G. Roelkens, D.V. Thourhout, S. Messaoudene, J.M. Fedeli, G.H. Duan, Low-Threshold Heterogeneously Integrated 
InP/SOI Lasers With a Double Adiabatic Taper Coupler, IEEE Photonics Technology Letters 24 (2012) 76-78.

[91] A. Abbasi, B. Moeneclaey, J. Verbist, X. Yin, J. Bauwelinck, G.H. Duan, G. Roelkens, G. Morthier, Direct and Electroabsorption Modulation of a III-V-on-Silicon DFB Laser at $56 \mathrm{~Gb} / \mathrm{s}$, IEEE Journal of Selected Topics in Quantum Electronics 23 (2017) 1-7.

[92] K. Tanabe, D. Guimard, D. Bordel, S. Iwamoto, Y. Arakawa, Electrically pumped $1.3 \mu \mathrm{m}$ roomtemperature InAs/GaAs quantum dot lasers on Si substrates by metal-mediated wafer bonding and layer transfer, Optics Express 18 (2010) 10604-10608.

[93] K. Tanabe, K. Watanabe, Y. Arakawa, III-V/Si hybrid photonic devices by direct fusion bonding, Scientific Reports 2 (2012) 349.

[94] K. Tanabe, T. Rae, K. Watanabe, Y. Arakawa, High-Temperature $1.3 \mu \mathrm{m}$ InAs/GaAs Quantum Dot Lasers on Si Substrates Fabricated by Wafer Bonding, Applied Physics Express 6 (2013) 082703.

[95] K. Tanabe, M. Nomura, D. Guimard, S. Iwamoto, Y. Arakawa, Room temperature continuous wave operation of InAs/GaAs quantum dot photonic crystal nanocavity laser on silicon substrate, Optics Express 17 (2009) 7036-7042.

[96] Y.H. Jhang, K. Tanabe, S. Iwamoto, Y. Arakawa, InAs/GaAs Quantum Dot Lasers on Silicon-onInsulator Substrates by Metal-Stripe Wafer Bonding, IEEE Photonics Technology Letters 27 (2015) 875878.

[97] P.J. Delfyett, D.H. Hartman, S.Z. Ahmad, Optical clock distribution using a mode-locked semiconductor laser diode system, Journal of Lightwave Technology 9 (1991) 1646-1649.

[98] J. Mandon, G. Guelachvili, N. Picqué, Fourier transform spectroscopy with a laser frequency comb, Nature Photonics 3 (2009) 99.

[99] N. Alic, S. Radic, in: OFC 2014, (2014).

[100] A.W. Fang, B.R. Koch, K.-G. Gan, H. Park, R. Jones, O. Cohen, M.J. Paniccia, D.J. Blumenthal, J.E. Bowers, A racetrack mode-locked silicon evanescent laser, Optics Express 16 (2008) 1393-1398.

[101] Z. Wang, M.L. Fanto, J.A. Steidle, A.A. Aboketaf, N.A. Rummage, P.M. Thomas, C.-S. Lee, W. Guo, L.F. Lester, S.F. Preble, Passively mode-locked InAs quantum dot lasers on a silicon substrate by PdGaAs wafer bonding, Applied Physics Letters 110 (2017) 141110.

[102] S. Keyvaninia, S. Uvin, M. Tassaert, Z. Wang, X. Fu, S. Latkowski, J. Marien, L. Thomassen, F. Lelarge, G. Duan, G. Lepage, P. Verheyen, J. Van Campenhout, E. Bente, G. Roelkens, III/V-on-silicon anti-colliding pulse-type mode-locked laser, Optics Letters 40 (2015) 3057-3060.

[103] V. Moskalenko, K.A. Williams, E.A.J.M. Bente, Integrated Extended-Cavity 1.5- $\mu$ m Semiconductor Laser Switchable Between Self- and Anti-Colliding Pulse Passive Mode-Locking Configuration, IEEE Journal of Selected Topics in Quantum Electronics 21 (2015) 40-45.

[104] K. Nozaki, T. Tanabe, A. Shinya, S. Matsuo, T. Sato, H. Taniyama, M. Notomi, Sub-femtojoule alloptical switching using a photonic-crystal nanocavity, Nature Photonics 4 (2010) 477.

[105] S. Matsuo, A. Shinya, T. Kakitsuka, K. Nozaki, T. Segawa, T. Sato, Y. Kawaguchi, M. Notomi, Highspeed ultracompact buried heterostructure photonic-crystal laser with $13 \mathrm{fJ}$ of energy consumed per bit transmitted, Nature Photonics 4 (2010) 648.

[106] C. Monat, C. Seassal, X. Letartre, P. Viktorovitch, P. Regreny, M. Gendry, P. Rojo-Romeo, G. Hollinger, E. Jalaguier, S. Pocas, B. Aspar, InP 2D photonic crystal microlasers on silicon wafer: room temperature operation at 1.55 \&mu;m, Electronics Letters 37 (2001) 764-766.

[107] G. Vecchi, F. Raineri, I. Sagnes, K.h. Lee, S. Guilet, L.L. Gratiet, F.V. Laere, G. Roelkens, D.V. Thourhout, R. Baets, A. Levenson, R. Raj, Photonic-crystal surface-emitting laser near $1.55 \mu \mathrm{m}$ on goldcoated silicon wafer, Electronics Letters 43 (2007) 39-40.

[108] T.J. Karle, Y. Halioua, F. Raineri, P. Monnier, R. Braive, L.L. Gratiet, G. Beaudoin, I. Sagnes, G. Roelkens, F.v. Laere, D.V. Thourhout, R. Raj, Heterogeneous integration and precise alignment of InPbased photonic crystal lasers to complementary metal-oxide semiconductor fabricated silicon-oninsulator wire waveguides, Journal of Applied Physics 107 (2010) 063103. 
[109] K. Takeda, T. Sato, A. Shinya, K. Nozaki, W. Kobayashi, H. Taniyama, M. Notomi, K. Hasebe, T. Kakitsuka, S. Matsuo, Few-fJ/bit data transmissions using directly modulated lambda-scale embedded active region photonic-crystal lasers, Nature Photonics 7 (2013) 569.

[110] K.A. Atlasov, M. Felici, K.F. Karlsson, P. Gallo, A. Rudra, B. Dwir, E. Kapon, 1D photonic band formation and photon localization in finite-size photonic-crystal waveguides, Optics Express 18 (2010) 117-122.

[111] G. Crosnier, D. Sanchez, S. Bouchoule, P. Monnier, G. Beaudoin, I. Sagnes, R. Raj, F. Raineri, Hybrid indium phosphide-on-silicon nanolaser diode, Nat Photon 11 (2017) 297-300.

[112] M.-K. Seo, K.-Y. Jeong, J.-K. Yang, Y.-H. Lee, H.-G. Park, S.-B. Kim, Low threshold current singlecell hexapole mode photonic crystal laser, Applied Physics Letters 90 (2007) 171122.

[113] T. Spuesens, F. Mandorlo, P. Rojo-Romeo, P. Regreny, N. Olivier, J.M. Fedeli, D.V. Thourhout, Compact Integration of Optical Sources and Detectors on SOI for Optical Interconnects Fabricated in a 200 mm CMOS Pilot Line, Journal of Lightwave Technology 30 (2012) 1764-1770.

[114] K.-Y. Jeong, Y.-S. No, Y. Hwang, K.S. Kim, M.-K. Seo, H.-G. Park, Y.-H. Lee, Electrically driven nanobeam laser, Nature Communications 4 (2013) 2822.

[115] J.V. Campenhout, P.R. Romeo, D.V. Thourhout, C. Seassal, P. Regreny, L.D. Cioccio, J.M. Fedeli, R. Baets, Design and Optimization of Electrically Injected InP-Based Microdisk Lasers Integrated on and Coupled to a SOI Waveguide Circuit, Journal of Lightwave Technology 26 (2008) 52-63.

[116] A.S.H. Liao, S. Wang, Semiconductor injection lasers with a circular resonator, Applied Physics Letters 36 (1980) 801-803.

[117] C. Seassal, P. Rojo-Romeo, X. Letartre, P. Viktorovitch, G. Hollinger, E. Jalaguier, S. Pocas, B. Aspar, InP microdisk lasers on silicon wafer: $\mathrm{CW}$ room temperature operation at 1.6 \&mu;m, Electronics Letters 37 (2001) 222-223.

[118] D. Liang, M. Fiorentino, T. Okumura, H.-H. Chang, D.T. Spencer, Y.-H. Kuo, A.W. Fang, D. Dai, R.G. Beausoleil, J.E. Bowers, Electrically-pumped compact hybrid silicon microring lasers for optical interconnects, Optics Express 17 (2009) 20355-20364.

[119] D. Liang, X. Huang, G. Kurczveil, M. Fiorentino, R.G. Beausoleil, Integrated finely tunable microring laser on silicon, Nature Photonics 10 (2016) 719.

[120] K. Mitsuo, U. Toshio, Molecular Beam Epitaxy of Controlled Single Domain GaAs on Si (100), Japanese Journal of Applied Physics 25 (1986) L285.

[121] K. Mitsuo, U. Toshio, Self-Annihilation of Antiphase Boundary in GaAs on Si(100) Grown by Molecular Beam Epitaxy, Japanese Journal of Applied Physics 26 (1987) L944.

[122] T.H. Windhorn, G.M. Metze, B.Y. Tsaur, J.C.C. Fan, AlGaAs double - heterostructure diode lasers fabricated on a monolithic GaAs/Si substrate, Applied Physics Letters 45 (1984) 309-311.

[123] T.H. Windhorn, G.M. Metze, Room - temperature operation of GaAs/AlGaAs diode lasers fabricated on a monolithic GaAs/Si substrate, Applied Physics Letters 47 (1985) 1031-1033.

[124] S. Sakai, T. Soga, M. Takeyasu, M. Umeno, Room - temperature laser operation of AlGaAs/GaAs double heterostructures fabricated on $\mathrm{Si}$ substrates by metalorganic chemical vapor deposition, Applied Physics Letters 48 (1986) 413-414.

[125] R. Fischer, W. Kopp, H. Morkoç, M. Pion, A. Specht, G. Burkhart, H. Appelman, D. McGougan, R. Rice, Low threshold laser operation at room temperature in $\mathrm{GaAs} /(\mathrm{Al}, \mathrm{Ga})$ As structures grown directly on (100)Si, Applied Physics Letters 48 (1986) 1360-1361.

[126] R.D. Dupuis, J.P.v.d. Ziel, R.A. Logan, J.M. Brown, C.J. Pinzone, Low - threshold high - efficiency AlGaAs - GaAs double - heterostructure injection lasers grown on Si substrates by metalorganic chemical vapor deposition, Applied Physics Letters 50 (1987) 407-409.

[127] D.G. Deppe, N.H. Jr., D.W. Nam, K.C. Hsieh, G.S. Jackson, R.J. Matyi, H. Shichijo, J.E. Epler, H.F. Chung, Room - temperature continuous operation of $p$ - $n$ AlxGa1-xAs - GaAs quantum well heterostructure lasers grown on Si, Applied Physics Letters 51 (1987) 637-639.

[128] D.G. Deppe, D.W. Nam, N.H. Jr., K.C. Hsieh, R.J. Matyi, H. Shichijo, J.E. Epler, H.F. Chung, Stability of $300 \mathrm{~K}$ continuous operation of $\mathrm{p}$ - n AlxGa1-xAs - GaAs quantum well lasers grown on $\mathrm{Si}$, Applied Physics Letters 51 (1987) 1271-1273. 
[129] J.P.v.d. Ziel, R.D. Dupuis, R.A. Logan, R.M. Mikulyak, C.J. Pinzone, A. Savage, Low threshold pulsed and continuous laser oscillation from AlGaAs/GaAs double heterostructures grown by metalorganic chemical vapor deposition on Si substrates, Applied Physics Letters 50 (1987) 454-456. [130] J.P.v.d. Ziel, R.D. Dupuis, R.A. Logan, C.J. Pinzone, Degradation of GaAs lasers grown by metalorganic chemical vapor deposition on Si substrates, Applied Physics Letters 51 (1987) 89-91.

[131] M. Razeghi, M. Defour, F. Omnes, P. Maurel, J. Chazelas, F. Brillouet, First GalnAsP - InP double - heterostructure laser emitting at $1.27 \mu \mathrm{m}$ on a silicon substrate, Applied Physics Letters 53 (1988) 725-727.

[132] M. Razeghi, M. Defour, R. Blondeau, F. Omnes, P. Maurel, O. Acher, F. Brillouet, J.C. C - Fan, J. Salerno, First cw operation of a Ga0.25In0.75As0.5P0.5 - InP laser on a silicon substrate, Applied Physics Letters 53 (1988) 2389-2390.

[133] T.C. Chong, C.G. Fonstad, Low - threshold operation of AlGaAs/GaAs multiple quantum well lasers grown on Si substrates by molecular beam epitaxy, Applied Physics Letters 51 (1987) 221-223. [134] M. Sugo, H. Mori, Y. Sakai, Y. Itoh, Stable cw operation at room temperature of a $1.5-\mu \mathrm{m}$ wavelength multiple quantum well laser on a Si substrate, Applied Physics Letters 60 (1992) 472-473. [135] M. Sugo, H. Mori, Y. Itoh, Y. Sakai, M. Tachikawa, $1.5 \mu \mathrm{m}$-Long-Wavelength Multiple Quantum Well Laser on a Si Substrate, Japanese Journal of Applied Physics 30 (1991) 3876.

[136] L. Cerutti, J.B. Rodriguez, E. Tournie, GaSb-Based Laser, Monolithically Grown on Silicon Substrate, Emitting at $1.55 \mu \mathrm{m}$ at Room Temperature, IEEE Photonics Technology Letters 22 (2010) 553-555.

[137] E. Tournié, L. Cerutti, J.-B. Rodriguez, H. Liu, J. Wu, S. Chen, Metamorphic III-V semiconductor lasers grown on silicon, MRS Bulletin 41 (2016) 218-223.

[138] Y. Arakawa, H. Sakaki, Multidimensional quantum well laser and temperature dependence of its threshold current, Applied Physics Letters 40 (1982) 939-941.

[139] T. Egawa, A. Ogawa, T. Jimbo, M. Umeno, AlGaAs/GaAs Laser Diodes with GaAs Islands Active Regions on Si Grown by Droplet Epitaxy, Japanese Journal of Applied Physics 37 (1998) 1552.

[140] K.K. Linder, J. Phillips, O. Qasaimeh, X.F. Liu, S. Krishna, P. Bhattacharya, J.C. Jiang, Self-organized In0.4Ga0.6As quantum-dot lasers grown on Si substrates, Applied Physics Letters 74 (1999) 1355-1357. [141] Z.I. Kazi, T. Egawa, M. Umeno, T. Jimbo, Growth of InxGa1-xAs quantum dots by metal-organic chemical vapor deposition on Si substrates and in GaAs-based lasers, Journal of Applied Physics 90 (2001) 5463-5468.

[142] J. Yang, P. Bhattacharya, Z. Mi, High-Performance $\operatorname{In}_{0.5} \mathrm{Ga}_{0.5} \mathrm{As} / \mathrm{GaAs}$ Quantum-Dot Lasers on Silicon With Multiple-Layer Quantum-Dot Dislocation Filters, IEEE Transactions on Electron Devices 54 (2007) 2849-2855.

[143] Z. Mi, J. Yang, P. Bhattacharya, G. Qin, Z. Ma, High-Performance Quantum Dot Lasers and Integrated Optoelectronics on Si, Proceedings of the IEEE 97 (2009) 1239-1249.

[144] Z. Mi, P. Bhattacharya, Pseudomorphic and Metamorphic Quantum Dot Heterostructures for Long-Wavelength Lasers on GaAs and Si, IEEE Journal of Selected Topics in Quantum Electronics 14 (2008) 1171-1179.

[145] M. Tang, S. Chen, J. Wu, Q. Jiang, V.G. Dorogan, M. Benamara, Y.I. Mazur, G.J. Salamo, A. Seeds, $\mathrm{H}$. Liu, 1.3- $\mu \mathrm{m}$ InAs/GaAs quantum-dot lasers monolithically grown on Si substrates using InAIAs/GaAs dislocation filter layers, Optics Express 22 (2014) 11528-11535.

[146] M. Tang, S. Chen, J. Wu, Q. Jiang, K. Kennedy, P. Jurczak, M. Liao, R. Beanland, A. Seeds, H. Liu, Optimizations of Defect Filter Layers for 1.3- $\mu \mathrm{m}$ InAs/GaAs Quantum-Dot Lasers Monolithically Grown on Si Substrates, IEEE Journal of Selected Topics in Quantum Electronics 22 (2016) 50-56.

[147] S. Chen, W. Li, J. Wu, Q. Jiang, M. Tang, S. Shutts, S.N. Elliott, A. Sobiesierski, A.J. Seeds, I. Ross, P.M. Smowton, H. Liu, Electrically pumped continuous-wave III-V quantum dot lasers on silicon, Nature Photonics 10 (2016) 307.

[148] M. Liao, S. Chen, S. Huo, S. Chen, J. Wu, M. Tang, K. Kennedy, W. Li, S. Kumar, M. Martin, T. Baron, C. Jin, I. Ross, A. Seeds, H. Liu, Monolithically Integrated Electrically Pumped Continuous-Wave 
III-V Quantum Dot Light Sources on Silicon, IEEE Journal of Selected Topics in Quantum Electronics 23 (2017) 1-10.

[149] H. Liu, T. Wang, Q. Jiang, R. Hogg, F. Tutu, F. Pozzi, A. Seeds, Long-wavelength InAs/GaAs quantum-dot laser diode monolithically grown on Ge substrate, Nat Photon 5 (2011) 416-419.

[150] A. Lee, Q. Jiang, M. Tang, A. Seeds, H. Liu, Continuous-wave InAs/GaAs quantum-dot laser diodes monolithically grown on Si substrate with low threshold current densities, Optics Express 20 (2012) 22181-22187.

[151] A.D. Lee, Q. Jiang, M. Tang, Y. Zhang, A.J. Seeds, H. Liu, InAs/GaAs Quantum-Dot Lasers Monolithically Grown on $\mathrm{Si}, \mathrm{Ge}$, and Ge-on-Si Substrates, IEEE Journal of Selected Topics in Quantum Electronics 19 (2013) 1901107-1901107.

[152] A.Y. Liu, C. Zhang, A. Snyder, D. Lubyshev, J.M. Fastenau, A.W.K. Liu, A.C. Gossard, J.E. Bowers, MBE growth of P-doped $1.3 \mu \mathrm{m}$ InAs quantum dot lasers on silicon, Journal of Vacuum Science \& Technology B, Nanotechnology and Microelectronics: Materials, Processing, Measurement, and Phenomena 32 (2014) 02 C108.

[153] A.Y. Liu, C. Zhang, J. Norman, A. Snyder, D. Lubyshev, J.M. Fastenau, A.W.K. Liu, A.C. Gossard, J.E. Bowers, High performance continuous wave $1.3 \mu \mathrm{m}$ quantum dot lasers on silicon, Applied Physics Letters 104 (2014) 041104.

[154] L. Shifren, R. Aitken, A.R. Brown, V. Chandra, B. Cheng, C. Riddet, C.L. Alexander, B. Cline, C. Millar, S. Sinha, G. Yeric, A. Asenov, Predictive Simulation and Benchmarking of Si and Ge pMOS FinFETs for Future CMOS Technology, IEEE Transactions on Electron Devices 61 (2014) 2271-2277.

[155] T. Wang, A. Lee, F. Tutu, A. Seeds, H. Liu, Q. Jiang, K. Groom, R. Hogg, The effect of growth temperature of GaAs nucleation layer on InAs/GaAs quantum dots monolithically grown on $\mathrm{Ge}$ substrates, Applied Physics Letters 100 (2012) 052113.

[156] Q. Jiang, M. Tang, S. Chen, J. Wu, A. Seeds, H. Liu, InAs/GaAs quantum-dot superluminescent diodes monolithically grown on a Ge substrate, Optics Express 22 (2014) 23242-23248.

[157] Y.W. Mo, D.E. Savage, B.S. Swartzentruber, M.G. Lagally, Kinetic pathway in Stranski-Krastanov growth of Ge on Si(001), Physical Review Letters 65 (1990) 1020-1023.

[158] D.J. Eaglesham, M. Cerullo, Dislocation-free Stranski-Krastanow growth of Ge on Si(100), Physical Review Letters 64 (1990) 1943-1946.

[159] H.-C. Luan, D.R. Lim, K.K. Lee, K.M. Chen, J.G. Sandland, K. Wada, L.C. Kimerling, High-quality Ge epilayers on Si with low threading-dislocation densities, Applied Physics Letters 75 (1999) 2909-2911. [160] A.Y. Liu, R.W. Herrick, O. Ueda, P.M. Petroff, A.C. Gossard, J.E. Bowers, Reliability of InAs/GaAs Quantum Dot Lasers Epitaxially Grown on Silicon, IEEE Journal of Selected Topics in Quantum Electronics 21 (2015) 690-697.

[161] A.Y. Liu, S. Srinivasan, J. Norman, A.C. Gossard, J.E. Bowers, Quantum dot lasers for silicon photonics [Invited], Photonics Research 3 (2015) B1-B9.

[162] N.G. Weimann, L.F. Eastman, D. Doppalapudi, H.M. Ng, T.D. Moustakas, Scattering of electrons at threading dislocations in GaN, Journal of Applied Physics 83 (1998) 3656-3659.

[163] R.J. Roedel, A.R. Von Neida, R. Caruso, L.R. Dawson, The Effect of Dislocations in Ga1 - x Al x As : Si Light - Emitting Diodes, Journal of The Electrochemical Society 126 (1979) 637-641.

[164] Wu J, Chen S, A. Seeds, H. Liu, Quantum dot optoelectronic devices: lasers, photodetectors and solar cells, J. Phys. D: Appl. Phys 48 (2015) 29.

[165] K. Böhm, B. Fischer, Photoluminescence at dislocations in GaAs and InP, Journal of Applied Physics 50 (1979) 5453-5460.

[166] R. Beanland, D.J. Dunstan, P.J. Goodhew, Plastic relaxation and relaxed buffer layers for semiconductor epitaxy, Advances in Physics 45 (1996) 87-146.

[167] T. Ward, A.M. Sánchez, M. Tang, J. Wu, H. Liu, D.J. Dunstan, R. Beanland, Design rules for dislocation filters, Journal of Applied Physics 116 (2014) 063508.

[168] I. George, F. Becagli, H.Y. Liu, J. Wu, M. Tang, R. Beanland, Dislocation filters in GaAs on Si, Semiconductor Science and Technology 30 (2015) 114004. 
[169] M. Tang, J. Wu, S. Chen, Q. Jiang, A.J. Seeds, H. Liu, V.G. Dorogan, M. Benamara, Y. Mazur, G. Salamo, in: IET Optoelectronics, (Institution of Engineering and Technology, 2015).

[170] B. Kunert, I. Németh, S. Reinhard, K. Volz, W. Stolz, Si (001) surface preparation for the antiphase domain free heteroepitaxial growth of GaP on Si substrate, Thin Solid Films 517 (2008) 140-143.

[171] R. Alcotte, M. Martin, J. Moeyaert, R. Cipro, S. David, F. Bassani, F. Ducroquet, Y. Bogumilowicz, E. Sanchez, Z. Ye, X.Y. Bao, J.B. Pin, T. Baron, Epitaxial growth of antiphase boundary free GaAs layer on $300 \mathrm{~mm}$ Si(001) substrate by metalorganic chemical vapour deposition with high mobility, APL Materials 4 (2016) 046101.

[172] Y. Wan, Q. Li, Y. Geng, B. Shi, K.M. Lau, InAs/GaAs quantum dots on GaAs-on-V-grooved-Si substrate with high optical quality in the $1.3 \mu \mathrm{m}$ band, Applied Physics Letters 107 (2015) 081106.

[173] Z. Wang, B. Tian, M. Pantouvaki, W. Guo, P. Absil, J. Van Campenhout, C. Merckling, D. Van Thourhout, Room-temperature InP distributed feedback laser array directly grown on silicon, Nat Photon 9 (2015) 837-842.

[174] I. Németh, B. Kunert, W. Stolz, K. Volz, Heteroepitaxy of GaP on Si: Correlation of morphology, anti-phase-domain structure and MOVPE growth conditions, Journal of Crystal Growth 310 (2008) 1595-1601.

[175] K. Volz, A. Beyer, W. Witte, J. Ohlmann, I. Németh, B. Kunert, W. Stolz, GaP-nucleation on exact Si (001) substrates for III/V device integration, Journal of Crystal Growth 315 (2011) 37-47.

[176] A.Y. Liu, J. Peters, X. Huang, D. Jung, J. Norman, M.L. Lee, A.C. Gossard, J.E. Bowers, Electrically pumped continuous-wave $1.3 \mu \mathrm{m}$ quantum-dot lasers epitaxially grown on on-axis (001) GaP/Si, Optics Letters 42 (2017) 338-341.

[177] D. Jung, Z. Zhang, J. Norman, R. Herrick, M.J. Kennedy, P. Patel, K. Turnlund, C. Jan, Y. Wan, A.C. Gossard, J.E. Bowers, Highly Reliable Low-Threshold InAs Quantum Dot Lasers on On-Axis (001) Si with 87\% Injection Efficiency, ACS Photonics (2017).

[178] D. Jung, P.G. Callahan, B. Shin, K. Mukherjee, A.C. Gossard, J.E. Bowers, Low threading dislocation density GaAs growth on on-axis GaP/Si (001), Journal of Applied Physics 122 (2017) 225703. [179] S. Chen, M. Liao, M. Tang, J. Wu, M. Martin, T. Baron, A. Seeds, H. Liu, Electrically pumped continuous-wave $1.3 \mu \mathrm{m}$ InAs/GaAs quantum dot lasers monolithically grown on on-axis Si (001) substrates, Optics Express 25 (2017) 4632-4639.

[180] Y. Wan, Q. Li, Y. Geng, B. Shi, K.M. Lau, InAs/GaAs quantum dots on GaAs-on-V-grooved-Si substrate with high optical quality in the $1.3 \mu \mathrm{m}$ band, Applied Physics Letters 107 (2015) 081106.

[181] J. Norman, M.J. Kennedy, J. Selvidge, Q. Li, Y. Wan, A.Y. Liu, P.G. Callahan, M.P. Echlin, T.M. Pollock, K.M. Lau, A.C. Gossard, J.E. Bowers, Electrically pumped continuous wave quantum dot lasers epitaxially grown on patterned, on-axis (001) Si, Optics Express 25 (2017) 3927-3934.

[182] Z. Wang, B. Tian, M. Paladugu, M. Pantouvaki, N. Le Thomas, C. Merckling, W. Guo, J. Dekoster, J. Van Campenhout, P. Absil, D. Van Thourhout, Polytypic InP Nanolaser Monolithically Integrated on (001) Silicon, Nano Letters 13 (2013) 5063-5069.

[183] B. Tian, Z. Wang, M. Pantouvaki, P. Absil, J. Van Campenhout, C. Merckling, D. Van Thourhout, Room Temperature O-band DFB Laser Array Directly Grown on (001) Silicon, Nano Letters 17 (2017) 559-564.

[184] B. Kunert, W. Guo, Y. Mols, B. Tian, Z. Wang, Y. Shi, D.V. Thourhout, M. Pantouvaki, J.V. Campenhout, R. Langer, K. Barla, III/V nano ridge structures for optical applications on patterned 300 mm silicon substrate, Applied Physics Letters 109 (2016) 091101.

[185] Y. Shi, Z. Wang, J. Van Campenhout, M. Pantouvaki, W. Guo, B. Kunert, D. Van Thourhout, Optical pumped InGaAs/GaAs nano-ridge laser epitaxially grown on a standard 300-mm Si wafer, Optica 4 (2017) 1468-1473.

[186] S.M. Chen, M.C. Tang, J. Wu, Q. Jiang, V.G. Dorogan, M. Benamara, Y.I. Mazur, G.J. Salamo, A.J. Seeds, H. Liu, $1.3 \mu \mathrm{m}$ InAs/GaAs quantum-dot laser monolithically grown on Si substrates operating over $100^{\circ} \mathrm{C}$ Electronics Letters 50 (2014) 1467-1468.

[187] T. Wang, H. Liu, A. Lee, F. Pozzi, A. Seeds, 1.3- $\mu \mathrm{m}$ InAs/GaAs quantum-dot lasers monolithically grown on Si substrates, Optics Express 19 (2011) 11381-11386. 
[188] S. Chen, M. Tang, Q. Jiang, J. Wu, V.G. Dorogan, M. Benamara, Y.I. Mazur, G.J. Salamo, P. Smowton, A. Seeds, H. Liu, InAs/GaAs Quantum-Dot Superluminescent Light-Emitting Diode Monolithically Grown on a Si Substrate, ACS Photonics 1 (2014) 638-642.

[189] J. Wu, A. Lee, Q. Jiang, M. Tang, A.J. Seeds, H. Liu, in: IET Optoelectronics, (Institution of Engineering and Technology, 2014).

[190] K.J. Vahala, Optical microcavities, Nature 424 (2003) 839.

[191] Q. Li, Y. Wan, A.Y. Liu, A.C. Gossard, J.E. Bowers, E.L. Hu, K.M. Lau, 1.3- $\mu \mathrm{m}$ InAs quantum-dot micro-disk lasers on V-groove patterned and unpatterned (001) silicon, Optics Express 24 (2016) 21038-21045.

[192] Y. Wan, Q. Li, A.Y. Liu, A.C. Gossard, J.E. Bowers, E.L. Hu, K.M. Lau, Optically pumped $1.3 \mu \mathrm{m}$ room-temperature InAs quantum-dot micro-disk lasers directly grown on (001) silicon, Optics Letters 41 (2016) 1664-1667.

[193] Y. Wan, Q. Li, A.Y. Liu, W.W. Chow, A.C. Gossard, J.E. Bowers, E.L. Hu, K.M. Lau, Sub-wavelength InAs quantum dot micro-disk lasers epitaxially grown on exact Si (001) substrates, Applied Physics Letters 108 (2016) 221101.

[194] N. Kryzhanovskaya, E. Moiseev, Y. Polubavkina, M. Maximov, M. Kulagina, S. Troshkov, Y. Zadiranov, Y. Guseva, A. Lipovskii, M. Tang, M. Liao, J. Wu, S. Chen, H. Liu, A. Zhukov, Heat-sink free $\mathrm{CW}$ operation of injection microdisk lasers grown on Si substrate with emission wavelength beyond $1.3 \mu \mathrm{m}$, Optics Letters 42 (2017) 3319-3322.

[195] Y. Wan, D. Jung, J. Norman, C. Shang, I. MacFarlane, Q. Li, M.J. Kennedy, A.C. Gossard, K.M. Lau, J.E. Bowers, O-band electrically injected quantum dot micro-ring lasers on on-axis (001) GaP/Si and Vgroove Si, Optics Express 25 (2017) 26853-26860.

[196] B. Shi, S. Zhu, Q. Li, Y. Wan, E.L. Hu, K.M. Lau, Continuous-Wave Optically Pumped $1.55 \mu \mathrm{m}$ InAs/InAlGaAs Quantum Dot Microdisk Lasers Epitaxially Grown on Silicon, ACS Photonics 4 (2017) 204-210.

[197] S. Zhu, B. Shi, Y. Wan, E.L. Hu, K.M. Lau, $1.55 \mu \mathrm{m}$ band low-threshold, continuous-wave lasing from InAs/InAlGaAs quantum dot microdisks, Optics Letters 42 (2017) 679-682.

[198] B. Shi, S. Zhu, Q. Li, C.W. Tang, Y. Wan, E.L. Hu, K.M. Lau, $1.55 \mu \mathrm{m}$ room-temperature lasing from subwavelength quantum-dot microdisks directly grown on (001) Si, Applied Physics Letters 110 (2017) 121109.

[199] J.M. Fedeli, F. Schrank, E. Augendre, S. Bernabé, J. Kraft, P. Grosse, T. Enot, Photonic-Electronic Integration With Bonding, IEEE Journal of Selected Topics in Quantum Electronics 20 (2014) 350-358. [200] V. Stojanović, R.J. Ram, M. Popović, S. Lin, S. Moazeni, M. Wade, C. Sun, L. Alloatti, A. Atabaki, F. Pavanello, N. Mehta, P. Bhargava, Monolithic silicon-photonic platforms in state-of-the-art CMOS SOI processes \&\#x0005B;Invited\&\#x0005D, Optics Express 26 (2018) 13106-13121.

[201] U. Yoshinori, N. Tatau, Epitaxial Lateral Overgrowth of GaAs on a Si Substrate, Japanese Journal of Applied Physics 28 (1989) L337. 